\title{
New galactic open cluster candidates from DSS and 2MASS imagery $\star, \star \star$
}

\author{
M. Kronberger ${ }^{1}$, P. Teutsch ${ }^{1,2}$, B. Alessi ${ }^{1}$, M. Steine ${ }^{1}$, L. Ferrero ${ }^{1}$, K. Graczewski ${ }^{1}$, M. Juchert ${ }^{1}$, D. Patchick ${ }^{1}$, \\ D. Riddle ${ }^{1}$, J. Saloranta ${ }^{1}$, M. Schoenball ${ }^{1}$, and C. Watson ${ }^{1}$
}

\author{
1 Deepskyhunters Collaboration \\ e-mail: deepskyhunters@yahoo.com \\ 2 Institut für Astrophysik, Leopold-Franzens-Universität Innsbruck, Austria \\ e-mail: Philipp.Teutsch@uibk.ac . at
}

Received 17 August 2005 / Accepted 5 October 2005

\section{ABSTRACT}

\begin{abstract}
An inspection of the DSS and 2MASS images of selected Milky Way regions has led to the discovery of 66 stellar groupings whose morphologies, color-magnitude diagrams, and stellar density distributions suggest that these objects are possible open clusters that do not yet appear to be listed in any catalogue. For 24 of these groupings, which we consider to be the most likely to be candidates, we provide extensive descriptions on the basis of 2MASS photometry and their visual impression on DSS and 2MASS. Of these cluster candidates, 9 have fundamental parameters determined by fitting the color-magnitude diagrams with solar metallicity Padova isochrones. An additional 10 cluster candidates have distance moduli and reddenings derived from $K$ magnitudes and $(J-K)$ color indices of helium-burning red clump stars. As an addendum, we also provide a list of a number of apparently unknown galactic and extragalactic objects that were also discovered during the survey.
\end{abstract}

Key words. Galaxy: open clusters and associations: general - HII regions - reflection nebulae - planetary nebulae: general

\section{Introduction}

In the most up-to-date catalogues of galactic star clusters, Dias et al. (2002) (hereafter DAML02) and Bica et al. (2003a) (hereafter BDB03), a total of 1875 open clusters, open cluster candidates, and stellar groups visible in the visual or infrared spectral bands are listed. Despite the large number of objects included in these catalogues, they are apparently still far from complete, illustrated by the fact that several hundred new clusters and cluster candidates have been added to the list of known stellar groupings since these catalogues were published.

This large number of new discoveries is mostly the result of the release of the Two Micron All Sky Survey (Skrutskie et al. 1997, hereafter 2MASS). The images and photometric data obtained from this survey in the $J, H$, and $K_{\mathrm{S}}$ spectral bands have proved to be fertile ground in the search for new open clusters and have offered new possibilities for the analysis of newly detected and already known cluster candidates. Important to mention in this respect are the following

\footnotetext{
* Figures 1-11 are only available in electronic form at http://www . edpsciences.org

$\star \star$ Tables 2e, 4a and $4 \mathrm{~b}$ are only, and Tables $2 \mathrm{a}-\mathrm{d}$ and 3 also, available in electronic form at the CDS via anonymous $\mathrm{ftp}$ to cdsarc.u-strasbg.fr $(130.79 .128 .5)$ or via

http://cdsweb.u-strasbg.fr/cgi-bin/qcat?J/A+A/447/921
}

publications: Bica et al. (2003c), who reports 3 new open cluster candidates in the Cygnus $\mathrm{X}$ region, detected by visually inspecting 2MASS and Digitized Sky Survey (hereafter DSS) images; Bica et al. (2003b) and Dutra et al. (2003), who carried out a survey of infrared star clusters and stellar groups around the central positions of optical and radio nebulae and found 167 and 179 previously unknown objects, respectively; and Ivanov et al. (2002) and Borissova et al. (2003), who used a search algorithm based on finding peaks in the apparent stellar surface density, leading to the detection of an additional 14 clusters via 2MASS.

Other important publications include: Alessi et al. (2003), who discovered 11 previously unknown cluster candidates in the solar neighborhood using astrometric and photometric data provided by the Tycho- 2 catalogue (Høg et al. 2000); Bica et al. (2004), who reported 3 new open clusters found on maps obtained from the Guide Star Catalogue and images from the DSS; and Drake (2005), who discovered 8 clusters by searching for density fluctuations within the USNO A2.0 catalogue.

The recent discovery of new galactic open clusters in optical wavelengths by Bica et al. (2003c, 2004) and Drake (2005) is remarkable in that as it illustrates that it is still possible to detect unknown clusters on the DSS, even though the systematic searches for faint clusters on the Palomar and ESO/SERC Schmidt plates - which led to the Berkeley open cluster list 
(Setteducati \& Weaver 1962) and to the individual lists which generated the ESO catalogue (Lauberts 1982) - were carried out very carefully. This is also underlined by Pfleiderer et al. (1977) and Saurer et al. (1994), who reported the discovery of a number of faint clusters apparently not detected during these surveys.

As a first result of a systematic survey of selected Milky Way fields based on the visual inspection of DSS and 2MASS images, we announce in this paper the discovery of 66 previously unknown (or, at least, unlisted) clusters and cluster candidates.

A discussion of the search technique and a description of the methods used to analyze the objects is given in the following section. Section 3 is dedicated to the presentation and discussion of the most interesting cluster candidates, and it provides a list of basic and fundamental parameters derived for these objects. Finally, in Sect. 4, concluding remarks are given.

\section{Search strategy and analysis}

A stellar field in which an open cluster is present is characterized by a number of specific features which reveal its existence:

1. The presence of an open cluster in a star field leads to increased stellar density when compared with the neighboring fields.

2. The cluster members show similar radial velocities and proper motions.

3. Due to their common origin, the member stars of an open cluster have similar ages and chemical compositions.

Starting with these considerations, we carried out a systematic survey of several Milky Way fields near the galactic plane using red, blue, and infrared First and Second Generation DSS images extracted from the ESO Online Digitized Sky Survey facility ${ }^{1}$. As target fields for this survey, we favored Milky Way regions close to the galactic equator and characterized by a distinct lack of catalogued open clusters, as well as fields with evidence of active star formation. The DSS images of these regions were then examined in order to search for fields containing aggregates with a morphology similar to open clusters, i.e. fields with a stellar density significantly higher than the surrounding ones. A number of reflection and emission nebulae visible on the DSS images were also investigated, and to examine whether they contained any embedded clusters or stellar groups, we extracted additional $J, H$, and $K_{\mathrm{S}}$ images of these objects from the 2MASS facility ${ }^{2}$. Finally, the exact position and apparent angular diameter of each cluster candidate were measured using the Aladin Interactive Sky Atlas provided by the CDS, Strasbourg ${ }^{3}$.

\subsection{Color-magnitude diagrams (CMDs)}

As an increased stellar density alone is not definite proof that the observed stellar field contains an open cluster (as it can

\footnotetext{
1 http://archive.eso.org/dss/dss

2 http://irsa.ipac.caltech.edu

3 http://aladin.u-strasbg.fr/aladin.gml
}

also be caused by chance agglomerations of stars and absorption holes), we obtained a photometric analysis of the candidate fields using data from the 2MASS Point Source Catalogue (Cutri et al. 2003) to get further clarity on their nature and their properties.

As a first step, we generated preliminary $[J,(J-H)]$ and $\left[K_{\mathrm{S}},\left(J-K_{\mathrm{S}}\right)\right] \mathrm{CMDs}$ for every candidate. To minimize possible contamination, we selected only those sources with accurate photometric measurements. This selection was done by checking the read flag $\left(r d_{-} f l g\right)$ of the sources provided in every band, which indicates how the magnitude of the source was determined and therefore contains information on the quality of the photometric measurement. Based on this criteria, we restricted the data base to: sources with $r d \_f l g=2$ (point spreadfunction fitting) or $r d \_f l g=1$ (aperture photometry); sources with a read flag of $0,4,6$, or 9 in any of the specific bands, indicating either that the source could not be detected in this band or that there was a bad photometric measurement, were omitted from the CMDs. In addition, we examined all other flags of the selected sources given in the 2MASS Point Source Catalogue, such as the quality flag ( $\left.p h \_q u a l\right)$ and the extended source contamination flag (gal_contam), to minimize the influence of further sources of error.

The CMDs of the cluster fields were then compared with the CMDs of 4 control fields with the same size as the cluster fields and situated N, S, E, and W of the cluster candidate; the center-to-center distance between comparison field and cluster field was, in each case, taken as 3 times the radius of the cluster candidate.

\subsection{Fundamental parameters}

The majority of the clusters and cluster candidates presented in this publication exhibited certain features in their CMDs which enabled us to determine fundamental parameters of these objects.

For those cluster candidates whose CMDs revealed a welldefined cluster sequence, we determined the distances, reddenings, and ages by fitting the cluster sequence with solar metallicity Padova isochrones from Girardi et al. (2002) calculated for the $J, H$, and $K_{\mathrm{S}}$ spectral bands ${ }^{4}$. To correct for the influence of interstellar reddening, we assumed a value of $R_{\mathrm{V}}=3.09$ for the total-to-selective absorption and used the relations $A_{\mathrm{J}}=0.282 \cdot A_{\mathrm{V}}$ and $E(J-H)=0.33 \cdot E(B-V)$ (Rieke $\&$ Lebowski 1985).

In a number of cases, the CMD exhibited a structure resembling a helium-burning red clump (hereafter RC). This RC - a typical feature of intermediate-aged and old open clusters - is a valuable standard candle and allows a determination of the clusters' distance and reddening. In case the CMD of such a cluster did not allow obtaining an isochrone fit, we applied the approach of Grocholski \& Sarajedini (2002, hereafter GS02), who used the mean values $m_{K, \mathrm{RC}}$ and $(J-K)_{\mathrm{RC}}$ of the stars composing the $\mathrm{RC}$ to derive the distance and reddening parameters. These values were computed by determining the

\footnotetext{
4 http://pleiadi.pd.astro.it/isoc_photosys.01/ isoc_photosys.01.html
} 
Table 1. mean values of $m_{K, \mathrm{RC}},(J-K)_{\mathrm{RC}}$ and $(J-K)_{\mathrm{RC}} /(J-H)_{\mathrm{RC}}$ for the clusters with RCs.

\begin{tabular}{lccc}
\hline \hline DSH ID & $m_{K, \mathrm{RC}}$ & $(J-K)_{\mathrm{RC}}$ & $(J-K)_{\mathrm{RC}} /(J-H)_{\mathrm{RC}}$ \\
\hline DSH J0347.3+5354 & $11.75 \pm 0.06$ & $1.50 \pm 0.03$ & $1.44 \pm 0.03$ \\
DSH J0920.5-5251 & $12.95 \pm 0.12$ & $1.12 \pm 0.01$ & $1.44 \pm 0.02$ \\
DSH J1054.2-6144 & $13.92 \pm 0.08$ & $1.09 \pm 0.03$ & $1.36 \pm 0.04$ \\
DSH J1323.6-6340 & $12.85 \pm 0.05$ & $1.11 \pm 0.01$ & $1.38 \pm 0.02$ \\
DSH J1906.8+0935 & $12.02 \pm 0.05$ & $1.66 \pm 0.02$ & $1.45 \pm 0.02$ \\
DSH J1920.8+1540 & $12.09 \pm 0.05$ & $1.72 \pm 0.01$ & $1.49 \pm 0.01$ \\
DSH J1940.1+2615 & $14.06 \pm 0.07$ & $1.06 \pm 0.03$ & $1.37 \pm 0.03$ \\
DSH J1942.7+2951 & $13.13 \pm 0.03$ & $0.90 \pm 0.03$ & $1.37 \pm 0.04$ \\
DSH J1945.1+2809 & $13.07 \pm 0.09$ & $0.91 \pm 0.01$ & $1.32 \pm 0.03$ \\
DSH J2126.1+5331 & $12.77 \pm 0.06$ & $1.40 \pm 0.02$ & $1.39 \pm 0.02$ \\
\hline
\end{tabular}

corresponding values $m_{K_{\mathrm{S}}, \mathrm{RC}}$ and $\left(J-K_{\mathrm{S}}\right)_{\mathrm{RC}}$ of the RC stars and subsequent transformation of the 2MASS $K_{\mathrm{S}}$ to the standard Johnson $K$ band magnitudes using the equations quoted in GS02 (see Table 1).

For the absolute magnitude $M_{K, \mathrm{RC}}$ of the RC, we adopted the value of $M_{K, \mathrm{RC}}=-1.61 \pm 0.03$ (Alves 2000) used in GS02. As color index $(J-K)_{0, \mathrm{RC}}$, we assumed $(J-K)_{0, \mathrm{RC}}=$ $0.62 \pm 0.05$, which was derived from solar metallicity Padova isochrones with $8.5 \leq \log t \leq 10.0$; this value is close to the mean value of $0.61 \pm 0.06$ for the clusters quoted in GS02.

To ensure that the observed feature is indeed an RC and not the brightest part of a highly reddened MS, we also computed the ratio $(J-K)_{\mathrm{RC}} /(J-H)_{\mathrm{RC}}$ (Table 1). Assuming moderate reddening $(0<E(B-V)<2)$, this ratio has a value of 1.2 to 1.4 for RC stars and a value close to 1.6 for stars of early spectral type; therefore, it provides information concerning the stellar composition of the feature.

It should be noted, however, that the parameters that were derived from $m_{K, \mathrm{RC}}$ and $(J-K)_{\mathrm{RC}}$ should only be taken as estimates, as both $M_{K, \mathrm{RC}}$ and $(J-K)_{0, \mathrm{RC}}$ show a dependence on age and metallicity (GS02; Girardi \& Salaris 2001); differences from the actual values of up to $30 \%$ can be expected. To determine more precise fundamental parameters, deeper studies of the cluster candidates are therefore required.

\subsection{Radial density profiles (RDPs)}

In addition to the CMDs, we created RDPs from 2MASS data by counting stars in concentric rings and dividing the counts by the area of each ring. To avoid misleading results due to spatial variations in the number of faint stars, we applied a cut-off at $H=15.5$ for optical clusters and at $K_{\mathrm{S}}=14.5$ for infrared clusters. These RDPs were used to determine the diameter of each candidate with good precision. They also provided a check on whether the fields of the potential clusters indeed exhibited a significant excess in the number of stars compared to the stellar background density.

\section{Results and discussion}

\subsection{Open clusters and promising open cluster candidates}

In Tables $2 \mathrm{a}$ to $2 \mathrm{c}$, we provide positions, angular diameters, reddenings, distances, and ages of 24 stellar aggregates visible in optical or infrared wavelengths that we consider as being open clusters or, at least, promising open cluster candidates according to the results of the analytical procedures described in Sect. 2. Cluster candidates whose CMDs allowed a fit to solar metallicity Padova isochrones are listed in Table 2a, while candidates with RCs in their CMDs are given in Table 2b. Finally, Table 2c contains those candidates for which the determination of fundamental parameters was found to be impossible. Every cluster candidate has a designation in the IAU-recognized format DSH Jhhmm.m \pm ddmm and its discoverer-ID assigned. Unless otherwise noted, all candidates were discovered between June 2003 and December 2004; the discovery was in each case announced to the DSH newsgroup ${ }^{5}$. One of the described cluster candidates (DSH J0718.1-1734 = Teutsch 49) turned out to be independently discovered by Drake (2005); it is listed therein as DC 2.

Figures 1 to 3 show the DSS images of all optical cluster candidates extracted from the CADC facility. The RDPs of the clusters are given in Figs. 4 to 6. Finally, in Figs. 7 to 9, the results of the photometric analysis of the clusters are presented. In each case, the radius of the extraction area equals the visually determined cluster radius $R_{\text {vis }}$. To increase the contrast between cluster features and background scatter in the CMDs, we used different grey-tones, with lighter tones indicating a larger distance from the cluster center.

\subsection{Extended notes}

\subsubsection{Isochrone-fitted cluster candidates}

DSH J0355.3+5823 = Juchert 9 is a small and slightly irregularly shaped cluster with 15 brighter stars and an unknown number of fainter members. It is well separated from the Milky Way background (Fig. 1). The center of the cluster is dominated by a pair of equally bright stars, which is listed in the Washington Catalogue of Double Stars (Worley \& Douglass 1997; hereafter WDS) as Stein 2015. As can be seen from the CMD shown in Fig. 7, the cluster stars align along a welldefined MS. A giant branch is not evident, implying that the cluster is a rather young object. As an upper limit, we found

DSH J0528.3+3446 = Kronberger 1 is an irregularly shaped, N-S elongated cluster situated $22^{\prime} \mathrm{N}$ of the HII region IC 417 and $4.7^{\prime} \mathrm{N}$ of the bright K0 star HD 35742 . The brightest cluster member, LS +34 33 (Reed 1998), is a luminous star of spectral type B, which is also listed as a double star with the designation Scheiner 272 in the WDS. The CMD of Kronberger 1 (Fig. 7) exhibits a sparsely populated, but fairly well-defined cluster sequence that lacks any evolutionary features and shows a gap between $J=11.5$ and $J=13$. The solar metallicity isochrone fit of this feature gives a distance of $d=1.9 \pm 0.2 \mathrm{kpc}$ and a reddening of $E(B-V)=0.52 \pm 0.06$. As in the case of Juchert 9, an exact age cannot be determined; the upper limit appears to be $\log t=7.5$.

The three cluster candidates DSH J0553.8+2649= Teutsch 51, DSH J1453.4-6028 = Teutsch 80 and

\footnotetext{
${ }^{5}$ http://groups.yahoo.com/group/deepskyhunters
} 
Table 2a. Positions, angular diameters, reddenings, distances, and ages of the open cluster candidates with CMDs fitted with Padova solar metallicity isochrones.

\begin{tabular}{llllcllll}
\hline \hline DSH ID & Discoverer ID & RA J2000 & Dec J2000 & $\begin{array}{c}D_{\text {vis }} \\
\text { arcmin }\end{array}$ & $E(B-V)$ & $(m-M)_{0}$ & $\begin{array}{c}\text { Distance } \\
\mathrm{kpc}\end{array}$ & $\begin{array}{c}\text { Age } \\
\log t\end{array}$ \\
\hline DSH J0355.3+5823 & Juchert 9 & 035521.0 & +582330 & 3.0 & $0.79 \pm 0.06$ & $13.20 \pm 0.30$ & $4.4 \pm 0.6$ & $<7.6$ \\
DSH J0528.3+3446 & Kronberger 1 & 052821.0 & +344630 & 1.6 & $0.52 \pm 0.06$ & $11.40 \pm 0.20$ & $1.9 \pm 0.2$ & $<7.5$ \\
DSH J0553.8+2649 & Teutsch 51 & 055351.9 & +264947 & 3.6 & $1.06 \pm 0.09$ & $12.60 \pm 0.20$ & $3.3 \pm 0.3$ & $8.90 \pm 0.10$ \\
DSH J1453.4-6028 & Teutsch 80 & 145325.6 & -602857 & 3.4 & $1.61 \pm 0.06$ & $11.95 \pm 0.60$ & $2.5 \pm 0.6$ & $8.10 \pm 0.15$ \\
DSH J1704.3-4204 & Teutsch 84 & 170420.1 & -420424 & 4.0 & $1.12 \pm 0.15$ & $11.75 \pm 0.25$ & $2.2 \pm 0.3$ & $9.00 \pm 0.10$ \\
DSH J1930.2+1832 & Teutsch 42 & 193013.1 & +183209 & 1.2 & $2.55 \pm 0.09$ & $11.00 \pm 0.20$ & $1.6 \pm 0.2$ & $7.50 \pm 0.25$ \\
DSH J1933.9+1831 & Kronberger 79 & 193355.0 & +183112 & 2.1 & $1.52 \pm 0.06^{a}$ & $12.80 \pm 0.30^{a}$ & $3.6 \pm 0.4^{a}$ & $7.40 \pm 0.30^{a}$ \\
& & & & & $1.30 \pm 0.06^{a}$ & $12.20 \pm 0.30^{a}$ & $2.7 \pm 0.4^{a}$ & $8.35 \pm 0.10^{a}$ \\
DSH J2002.3+3518 & ADS 13292 Cl & 200223.3 & +351841 & 1.1 & $0.58 \pm 0.03$ & $11.00 \pm 0.20$ & $1.6 \pm 0.2$ & $<7.0$ \\
DSH J2027.7+3604 & Teutsch 30 & 202743.0 & +360432 & 3.2 & $1.24 \pm 0.03$ & $11.05 \pm 0.15$ & $1.6 \pm 0.1$ & $<6.9$ \\
\hline
\end{tabular}

${ }^{a}$ See Sect. 3.2.1.

Table 2b. Positions, angular diameters, reddenings and distances of the open cluster candidates with RCs.

\begin{tabular}{lllllcrr}
\hline \hline DSH ID & Discoverer ID & RA J2000 & Dec J2000 & $\begin{array}{c}D_{\text {vis }} \\
\text { arcmin }\end{array}$ & $E(B-V)$ & $(m-M)_{0}$ & $\begin{array}{c}\text { Distance } \\
\mathrm{kpc}\end{array}$ \\
\hline DSH J0347.3+5354 & Juchert 11 & 034718.0 & +535435 & 5.0 & $1.68 \pm 0.15$ & $12.75 \pm 0.15$ & $3.6 \pm 0.2$ \\
DSH J0920.5-5251 & Teutsch 48 & 092031.8 & -525106 & 2.2 & $0.96 \pm 0.11$ & $14.25 \pm 0.20$ & $7.0 \pm 0.6$ \\
DSH J1054.2-6144 & Kronberger 39 & 105413.6 & -614416 & 0.8 & $0.90 \pm 0.15$ & $15.20 \pm 0.15$ & $11.1 \pm 0.8$ \\
DSH J1323.6-6340 & Teutsch 79 & 132338.8 & -634010 & 2.0 & $0.95 \pm 0.12$ & $14.15 \pm 0.10$ & $6.7 \pm 0.4$ \\
DSH J1906.8+0935 & Alessi 56 & 190652.1 & +093500 & 2.2 & $2.00 \pm 0.13$ & $12.95 \pm 0.10$ & $3.9 \pm 0.2$ \\
DSH J1920.8+1540 & Alessi 57 & 192053.8 & +154036 & 2.5 & $2.11 \pm 0.11$ & $12.95 \pm 0.10$ & $3.9 \pm 0.2$ \\
DSH J1940.1+2615 & Kronberger 31 & 194011.0 & +261548 & 1.3 & $0.84 \pm 0.15$ & $15.40 \pm 0.15$ & $11.9 \pm 0.8$ \\
DSH J1942.7+2951 & Teutsch 43 & 194246.9 & +295120 & 1.3 & $0.54 \pm 0.15$ & $14.55 \pm 0.10$ & $8.1 \pm 0.4$ \\
DSH J1945.1+2809 & Kronberger 4 & 194511.4 & +280940 & 1.3 & $0.56 \pm 0.12$ & $14.50 \pm 0.15$ & $7.9 \pm 0.6$ \\
DSH J2126.1+5331 & Kronberger 81 & 212608.9 & +533158 & 3.5 & $1.50 \pm 0.13$ & $13.85 \pm 0.15$ & $5.9 \pm 0.4$ \\
\hline
\end{tabular}

Table 2c. Positions and angular diameters of open cluster candidates without fundamental parameters determined.

\begin{tabular}{lllcc}
\hline \hline DSH ID & Discoverer ID & RA J2000 & Dec J2000 & $\begin{array}{c}D_{\text {vis }} \\
\text { arcmin }\end{array}$ \\
\hline DSH J0718.4-1734 & Teutsch 49 & 071826.8 & -173440 & 2.4 \\
DSH J1052.8-5927 & Teutsch 31 & 105250.1 & -592753 & 1.0 \\
DSH J1822.6-1443 & Kronberger 25 & 182239.9 & -144341 & 1.0 \\
DSH J2003.1+3158 & Kronberger 54 & 200307.9 & +315801 & 0.9 \\
DSH J2111.8+5222 & Kronberger 80 & 211150.5 & +522248 & 0.9 \\
\hline
\end{tabular}

DSH J1704.3-4204 = Teutsch 84 are cluster candidates that are situated in Milky Way parts that appear to be heavily obscured by interstellar dust; it is therefore possible that these objects are actually regions of lower extinction and not physical groups of stars. Nonetheless, in all cases the CMD (Fig. 7) supports the idea of a real clustering, as they all reveal well-defined structures that resemble cluster sequences and are not visible in the CMDs of the comparison fields.

DSH J1930.2+1832 = Teutsch 42 is a compact cluster candidate that is located $17^{\prime} \mathrm{N}$ of the HII region S 82 (Sharpless 1959 ) in Sagitta. It appears as a tight group dominated by a triangle of brighter stars (Fig. 1). As its color excess of $E(B-V)=$ $2.55 \pm 0.09$ implies, it is a highly reddened object. One of the remarkable things about this cluster is the presence of the X-ray source 2E 1928.0+1825 = 2RXP J193013.8+183216 (Harris et al. 1994; ROSAT 2000) in its field. Whether there is indeed a physical connection between this object and the cluster requires deeper study and will not be discussed in this publication; we note, however, the short distance from the cluster center of $12 \pm 10 \operatorname{arcsec}($ ROSAT 2000).

DSH J1933.9+1831 = Kronberger 79 is a compact object of irregular morphology that contrasts very well with the Milky Way background (Fig. 1). This is also indicated by its RDP (Fig. 4). With a sharp central peak and a steady decline with increasing distance from the center, it has a shape that is typical of open clusters with a strong central condensation. Photometric analysis of the cluster field reveals a well defined cluster sequence with a MS, a possible turn-off at about $J=12$ 
Table 2d. Positions and angular diameters of additional open cluster candidates.

\begin{tabular}{|c|c|c|c|c|c|}
\hline DSH ID & Discoverer ID & RA J2000 & Dec J2000 & $\begin{array}{c}D_{\text {vis }} \\
\text { arcmin }\end{array}$ & Notes \\
\hline DSH J0016.3+5957 & Juchert-Saloranta 1 & 001620.5 & +595743 & 5.0 & \\
\hline DSH J0033.1+6507 & Patchick 78 & 003310.2 & +650703 & 1.8 & \\
\hline DSH J0207.3+6015 & Riddle 4 & 020722.7 & +601525 & 4.0 & brightest star HD 236946 (spectrum K2; Brodskaya 1958) probable non-member \\
\hline DSH J0247.7+6158 & Teutsch 162 & 024742.3 & +615829 & 1.7 & [BDS2003] 57 (Bica et al. 2003b) 2.5' SW; involved in Sh2-193 (Sharpless 1959) \\
\hline DSH J0455.7+3646 & Teutsch 5 & 045543.2 & +364655 & 3.2 & \\
\hline DSH J0539.4+3320 & Teutsch 1 & 053929.3 & +332046 & 2.0 & \\
\hline DSH J0541.3+3914 & Teutsch 2 & 054121.8 & +391426 & 1.4 & PN G170.7+04.6 (Alter et al. 1970) 42" distant from the cluster center \\
\hline DSH J0544.3+2848 & Teutsch 10 & 054423.0 & +284854 & 5.5 & situated within the NE part of Sh2-240 (Sharpless 1959) \\
\hline DSH J0604.1+3129 & Kronberger 60 & 060410.1 & +312944 & 1.8 & IR cluster or group; embedded in reflection nebula GN 06.00.8 (Magakian 2003) \\
\hline DSH J0625.4+1351 & Teutsch 11 & 062524.4 & +135159 & 2.3 & DSH J0625.6+1336 = Teutsch $1216^{\prime} \mathrm{S}$ \\
\hline DSH J0625.6+1336 & Teutsch 12 & 062540.3 & +133625 & 4.2 & DSH J0625.4+1351 = Teutsch $1116^{\prime} \mathrm{N}$ \\
\hline DSH J0629.4+0910 & Alessi 53 & 062924.5 & +091039 & 2.8 & $=$ DC $7($ Drake 2005) \\
\hline DSH J0643.0+0140 & Alessi 15 & 064304.0 & +014019 & 0.9 & DSH J0643.9+0124= Teutsch 13 20.6' SE; DSH J0643.5+0210 = Alessi 16 31' NNE \\
\hline DSH J0643.5+0210 & Alessi 16 & 064335.0 & +021024 & 0.6 & embedded in nebulosity; DSH J0643.0+0140 = Alessi $1531^{\prime}$ SSW \\
\hline DSH J0643.9+0124 & Teutsch 13 & 064355.0 & +012409 & 2.5 & DSH J0643.0+0140 = Alessi $1520.6^{\prime} \mathrm{NW}$ \\
\hline DSH J0720.9-2251 & Juchert 12 & 072056.7 & -225200 & 2.8 & 1.4' SE of HD 57573 \\
\hline DSH J0758.3-3446 & Kronberger 85 & 075820.8 & -344611 & 1.4 & 1.6' NW of reflection nebula Bran 89 (Magakian 2003) \\
\hline DSH J1108.6-6042 & Teutsch $143 \mathrm{a}$ & 110840.6 & -604250 & 0.6 & surrounding S Dor star Wray 15-751 (van Genderen 2001) \\
\hline DSH J1153.2-6236 & Teutsch 77 & 115315.5 & -623632 & 1.5 & HD $3092052.8^{\prime}$ NNE \\
\hline DSH J1713.2-3942 & Teutsch 85 & 171313.8 & -394222 & 0.6 & \\
\hline DSH J1907.5+0617 & Juchert 3 & 190733.0 & +061710 & 3.0 & \\
\hline DSH J1911.1+1450 & Riddle 15 & 191109.2 & +145004 & 0.8 & partly embedded in nebulosity \\
\hline DSH J1922.5+1240 & Juchert 1 & 192232.0 & +124000 & 3.2 & at N edge of LDN 684 (Lynds 1962) \\
\hline DSH J1925.2+1356 & Kronberger 13 & 192514.9 & +135644 & 1.5 & C1922+136 = King $2518^{\prime}$ SW; DSH J1926.0+1945 = Teutsch $2616^{\prime}$ SE \\
\hline DSH J1937.3+1841 & Teutsch 27 & 193723.2 & +184150 & 1.1 & DSH J1933.9+1831 = Kronberger 79 53' WSW \\
\hline DSH J1958.1+3053 & Kronberger 52 & 195807.9 & +305318 & 2.3 & $8.6^{\prime} \mathrm{SW}$ of HD 189395 \\
\hline DSH J1959.5+4918 & Patchick 89 & 195933.0 & +491845 & 1.4 & $=$ DC $6($ Drake 2005$)$ \\
\hline DSH J2001.2+3336 & Toepler 1 & 200117.6 & +333654 & 2.1 & HII region M 1-97 (Minkowski 1946) 3.3' SE; see also Toepler (1998) \\
\hline DSH J2006.5+3534 & Kronberger 28 & 200631.7 & +353434 & 1.3 & in field of C2004+356 = NGC 6871 \\
\hline DSH J2015.0+3355 & Patchick 75 & 201501.5 & +335543 & 1.5 & \\
\hline DSH J2017.9+3645 & Kronberger 74 & 201757.2 & +364537 & 0.3 & IR cluster or group; embedded in nebulosity; HII region Sh2-104 (Sharpless 1959) W \\
\hline DSH J2020.7+4112 & Kronberger 58 & 202047.8 & +411217 & 0.2 & IR cluster or group; embedded in nebulosity; V1318 Cyg cluster $10^{\prime} \mathrm{NNW}$ \\
\hline DSH J2023.9+3636 & Kronberger 57 & 202357.6 & +363617 & 2.8 & \\
\hline DSH J2028.2+3506 & Teutsch 28 & 202816.5 & +350650 & 1.3 & \\
\hline DSH J2033.8+4008 & Kronberger 59 & 203349.9 & +400853 & 1.6 & IR cluster or group; embedded in nebulosity; associated with IRAS 20319+3958 \\
\hline DSH J2035.1+5318 & Patchick 103 & 203508.3 & +531800 & 1.3 & \\
\hline DSH J2353.1+6247 & Kronberger 55 & 235309.5 & +624712 & 1.0 & IR cluster \\
\hline
\end{tabular}

and $(J-H)=0.4$, and a few possible giant stars positioned around $J=11.2$ and $(J-H)=0.9$ (Fig. 7). Assuming that these stars are indeed members of Kronberger 79, distance, age, and color excess are found to be $d=2.7 \pm 0.4 \mathrm{kpc}$, $E(B-V)=1.30 \pm 0.06$, and $\log t=8.35 \pm 0.1$. Another solution, excluding the giant stars, yields $d=3.6 \pm 0.4 \mathrm{kpc}$, $E(B-V)=1.52 \pm 0.06$, and $\log t=7.4 \pm 0.3$.

DSH J2002.3+3518 = ADS $13292 \mathrm{Cl}$ is a very compact group with a morphology similar to the Trapezium Cluster in the Orion Nebula (Fig. 1). Although it was noted as a multiple star as early as 1894 by Thomas Espin, who catalogued it as Espin 202 (WDS), and listed as potential trapezium system \#67 by Ambartsumian (1954), it was not recognized as a possible cluster until recently when it was independently discovered by B. Skiff and P. Teutsch (Archinal \& Hynes 2003). A recent study of trapezium systems by Abt \& Corbally (2000) (hereafter AC00) revealed the group indeed to be a physical system and identified 6 probable cluster members, all of them within $0.55^{\prime}$ from the cluster center. However, as AC00 considered just the brightest stars of the system and ignored the fainter group members the actual number of cluster members may therefore be much higher. From a solar metallicity isochrone fit of the CMD (Fig. 7), we found a distance modulus $(m-M)_{0}$ of $11.0 \pm 0.2$ and a color excess $E(B-V)=0.58 \pm 0.06$. This is in good agreement with the values quoted in AC00, which are $(m-M)_{0}=10.9$ and $E(B-V)=0.61$, respectively. The cluster appears to be a very young object, with an age of probably less than $10 \mathrm{Myr}$.

DSH J2027.7+3604 = Teutsch 30 is a fairly poor and loose group of stars associated with the faint HII region LBN 198 (Lynds 1965) and located immediately E of a long, thin streak of interstellar matter that runs in $\mathrm{N}-\mathrm{S}$ direction (Fig. 1). The stellar aggregate is positioned around the B star BD+35 4126, which is also listed in the WDS as a quadruple star with the designation Espin 2193. As its position within a HII region suggests, this cluster appears to be a very young object, with $\log t \leq 6.9$.

\subsubsection{Cluster candidates with RCs}

DSH J1323.6-6340 = Teutsch 79 is a faint cloud of stars not well separated from the background Milky Way and with an obvious dark spot in the immediate center (Fig. 2). Its CMD (Fig. 8) reveals a distinct RC with similarities to the structure that is observed in the CMD of the cluster 
C $1426-607=$ Pismis 19 (DAML02) (Fig. 10). From the position of the $\mathrm{RC}$ in the $\mathrm{CMD}$, distance and reddening of this cluster candidate are found to be $d=6.7 \pm 0.4 \mathrm{kpc}$ and $E(B-V)=0.95 \pm 0.12$.

DSH $\mathbf{J} 2126.1+5331=$ Kronberger 81 is a rich cluster of stars, appearing as a dense cloud of extremely faint stars on red and infrared DSS images (Fig. 2); on blue DSS images, the cluster is invisible, indicating strong interstellar absorption in the cluster field. As in the case of Teutsch 42, an X-ray source is located in the immediate vicinity of the cluster, about $2.0^{\prime}$ distant from the cluster center and less than 10" from the star TYC 3966-892-1; its designation in the ROSAT all-sky Bright Source Catalogue (Voges et al. 1999) is 1RXS J212622.1+533224. The CMD of the cluster (Fig. 8) differs significantly from the CMDs of the comparison fields, as it completely lacks a distinct population of blue stars but instead reveals a conspicuous feature on the red side of the diagram at $(J-H) \approx 1$, which is, in all probability, the well-populated giant branch of the cluster, with the RC positioned at $J \approx 14$. This is especially distinct in the innermost $0.55^{\prime}$ of the cluster, where almost all stars detected by 2 MASS align along this feature. All this is a strong argument in favor of the reality of this object, as the presence of a dust hole, which might have a similar optical appearance, would result in a shift of the stellar distribution to the blue side of the CMD. Moreover, the $(J-K)_{\mathrm{RC}} /(J-H)_{\mathrm{RC}}$ value of $1.39 \pm 0.02$ that is determined for this feature (see Table 1) indicates that it is indeed composed of giant stars and not a population of reddened main-sequence stars. As a comparison with the cluster NGC 2158 (whose giant branch is of comparable appearance) shows, the lack of an MS in the CMD of Kronberger 81 is probably due to the fact that the MS stars are too faint to be detected (Fig. 11). Assuming that both objects have similar properties in respect to age and stellar composition, the brightness of the turn-off can be estimated as $\approx 16$ in $J$ band and would therefore be at the limit of detection for 2MASS. As follows from $m_{K, \mathrm{RC}}$ and $(J-K)_{\mathrm{RC}}$, distance and reddening of Kronberger 81 are found to be $d=5.9 \pm 0.4 \mathrm{kpc}$ and $E(B-V)=1.50 \pm 0.13$; like several other clusters presented in this paper, it appears to be a member of the Perseus Arm of the Galaxy.

The cluster candidates DSH J0347.3+5354 = Juchert 11, DSH J0920.5-5251 = Teutsch 48, DSH J1054.2-6144 = Kronberger 39, DSH J1906.8+0935 = Alessi 56, DSH J1920.8+1540 = Alessi 57, DSH J1940.1+2615 = Kronberger 31, DSH J1942.7+2951 = Teutsch 43, and DSH J1945.1+2809 = Kronberger 4 all appear as faint but distinct concentrations of stars (Fig. 2), and all have similar CMDs with distinct clumps of red giant stars present that are not evident in the CMDs of the comparison fields (Fig. 8). The RDPs (Fig. 5) are in all cases consistent with the assumption of an open cluster in the field, but most of them lack the very distinct over-density of stars evident in the RDPs of the majority of the cluster and cluster candidates presented here due to the faintness of the majority of stars composing these aggregates, which puts them below the cut-off we defined in Sect. 2. The distances $d$ of these clusters vary between $3.6 \pm 0.2 \mathrm{kpc}$ for Juchert 11 and $11.9 \pm 0.8 \mathrm{kpc}$ for Kronberger 31 while reddenings $E(B-V)$ are between $0.54 \pm 0.15$ (Teutsch 43) and $2.11 \pm 0.11$ (Alessi 57).

\subsubsection{Cluster candidates without fundamental parameters}

DSH J0718.1-1734 = Teutsch 49 is a fairly rich cluster of faint stars that is separated very well from the Milky Way background (Fig. 3). It is identical with the object DC 2 in Drake (2005). The visually estimated diameter of this cluster is $2.4^{\prime}$, but the RDP (Fig. 6) suggests that outliners extend as far as $2.2^{\prime}$ from the cluster center. A similar cluster radius $\left(2.4^{\prime}\right)$ is found by Drake (2005), who furthermore notes a King model core radius of $0.55^{\prime}$ for this cluster. The cluster CMD (Fig. 9) reveals a well-defined MS at $(J-H) \approx 0.6$, which implies strong reddening $E(B-V)$ in the range of 1.5-2.0. A giant branch is not evident in the CMD, indicating that the stellar aggregate is rather young.

DSH J1052.8-5927 = Teutsch 31 is a very compressed starcluster candidate with a diameter of 1.2 arcmin in the vicinity of the $\eta$ Carinae Nebula NGC 3372. The CMD of this candidate (Fig. 9) contrasts well with the comparison fields and reveals traces of the MS at about $(J-H)=0.4$, but it is not defined well enough to determine any fundamental parameters.

DSH J1822.6-1443 = Kronberger 25 appears as an extremely dense cluster of stars in the field of the HII region $\mathrm{S} 48$ (Sharpless 1959), with the clusters C 1819-146 and C 1820-146 (Kharchenko \& Schilbach 1995; hereafter KS95) situated at distances of 10 and 5.7 arcmin due NW and N, respectively. The tightly packed central core of the cluster measures about 20 arcsec across and contains at least 8 stars, while the overall diameter of the group appears to be about $1^{\prime}$ (Fig. 3).

Due to the high stellar density in the cluster center, which causes the photometric measurements of the inner most stars to be greatly influenced by blending, the CMD (Fig. 9) is illdefined, with just traces of the MS visible. For that reason, a direct determination of the cluster parameters was impossible. However, it has to be noted that Kronberger 25 is shown in Fig. 12 of KS95 (therein dotted as a single star) to share the proper motion of the nearby cluster complex; this implies that this newly discovered cluster might in fact be associated with this complex and, therefore, be situated at a similar distance $\left(d_{\mathrm{C} 1819-146}=1.99 \mathrm{kpc} ; d_{\mathrm{C} 1820-146}=2.13 \mathrm{kpc}\right.$; from KS95).

DSH J2003.1+3158 = Kronberger 54 is a small cluster of stars that is well separated from the Milky Way background. It is situated 4.1' $\mathrm{E}$ of the bright star HD 190227 and 3.6' $\mathrm{N}$ of the possible planetary nebula CoMaC 3 (Acker et al. 1992).

The CMD of Kronberger 54 (Fig. 9) shows practically all cluster stars aligned along a well-defined, although fairly broad MS; a giant branch is not present, indicating that this group is of rather young age. Unfortunately, the scatter of the MS, together with the obvious lack of stars with $J>15$ in the cluster field, prevents a reliable determination of the fundamental parameters of this cluster. 
Table 3. Positions and angular diameters of apparently uncatalogued galactic and extragalactic objects.

\begin{tabular}{|c|c|c|c|c|c|c|}
\hline DSH ID & Discoverer ID & RA J2000 & Dec J2000 & $\begin{array}{c}D_{\max } \\
\text { arcmin }\end{array}$ & $\begin{array}{c}D_{\min } \\
\operatorname{arcmin}\end{array}$ & Notes \\
\hline DSH J0540.7+3144 & Teutsch PN J0540.7+3144 & 054044.6 & +314432 & 1.8 & 1.8 & PN; independent IPHAS discovery (Frew 2005d) \\
\hline DSH J0542.7+3057B & Teutsch GN J0542.7+3057 & 054244.3 & +305757 & 2.1 & 2.1 & HII region? Associated with DSH J0542.7+3057A = Teutsch 45 \\
\hline DSH J0600.5+2141 & Teutsch GN J0600.5+2141 & 060034.8 & +214111 & 1.2 & 1.1 & compact $\mathrm{HII}$ region or PN \\
\hline DSH J0646.4+0828 & Riddle PN J0646.4+0828 & 064624.7 & +082902 & 1.1 & 1.0 & confirmed spectroscopically as PN (Frew 2005a) \\
\hline DSH J1405.9-6040 & Riddle GN J1405.9-6040 & 140558.5 & -604014 & 1.7 & 1.1 & reflection nebula? \\
\hline DSH J1714.8-1415 & Patchick 2 & 171448.9 & -141554 & 0.17 & 0.17 & small disk $0.6^{\prime} \mathrm{N}$ of SAO 160398 ; planetary nebula? \\
\hline DSH J1909.9+1204 & Teutsch GN J1909.9+1204 & 190954.9 & +120452 & 0.2 & 0.2 & reddish circular nebulosity; planetary nebula? \\
\hline DSH J1919.5+4445 & Patchick 5 & 191930.6 & +444544 & 2.6 & 1.6 & probable bipolar PN (Block 2005; Frew 2005d) \\
\hline DSH J1940.6+2930 & Kronberger PN J1940.6+2930 & 194040.4 & +293009 & 0.4 & 0.4 & misclass. as galaxy by Roman et al. (2000); PN (Frew 2005d) \\
\hline DSH J1941.1+1908 & Kronberger GN J1941.1+1908 & 194107.7 & +190826 & 0.6 & 0.3 & irregular nebulosity surrounding 2 stars; classification uncertain \\
\hline DSH J1942.4+2145 & Kronberger PN J1942.4+2145 & 194226.1 & +214523 & 0.35 & 0.3 & small bipolar nebulosity; PN? \\
\hline DSH J1944.9+2245 & Kronberger PN J1944.9+2245 & 194459.1 & +224549 & 4.0 & 4.0 & PN (Frew 2005d); Lan 21 (Lanning \& Meakes 2000) assoc. \\
\hline DSH J1947.0+2930 & Patchick 1 & 194702.7 & +293026 & 0.21 & 0.18 & confirmed spectroscopically as PN (Frew 2005d) \\
\hline DSH J1957.3+2639 & Teutsch PN J1957.3+2639 & 195722.3 & +263906 & 2.3 & 1.5 & confirmed spectroscopically as PN (Frew 2005c); bipolar? \\
\hline DSH J2002.4+5533 & Patchick 4 & 200228.4 & +553318 & 3.5 & 3.5 & nebulosity with very blue central star; PN? (Frew 2005d) \\
\hline DSH J2009.6+4114 & Patchick 6 & 200940.9 & +411443 & 0.7 & 0.7 & probable PN (Frew 2005d) \\
\hline DSH J2046.1+5257 & Patchick 3 & 204610.5 & +525706 & 0.5 & 0.5 & nebulosity with very blue central star; planetary nebula? \\
\hline DSH J2051.0+7222 & Schoenball GN J2051.0+7222 & 205105.0 & +722227 & 2.5 & 2.0 & evenly illuminated elliptical nebula; reflection nebula? \\
\hline DSH J2055.4+3903 & Teutsch PN J2055.4+3903 & 205527.3 & +390357 & 0.35 & 0.25 & small bipolar nebulosity; planetary nebula? \\
\hline DSH J2120.0+5141 & Patchick 7 & 212000.1 & +514105 & 0.08 & 0.08 & very small emission-line object; planetary nebula? \\
\hline DSH J2122.0+5504 & Kronberger PN J2122.0+5504 & 212201.0 & +550430 & 0.33 & 0.33 & nebulosity consisting of 2 opposing arcs; planetary nebula? \\
\hline DSH J2308.8+1712 & Riddle GX J2308.8+1712 & 230851.4 & +171236 & 1.8 & 1.5 & bright galaxy; variable star DY Pegasi superimposed \\
\hline DSH J2327.2+6509 & Teutsch PN J2327.2+6509 & 232713.8 & +650915 & 0.3 & 0.3 & probable planetary nebula (Frew 2005b) \\
\hline
\end{tabular}

DSH J2111.8+5222 = Kronberger 80 is also a likely galactic cluster. It is situated in a part of the Milky Way with fairly strong interstellar obscuration. Very small $\left(D_{\text {vis }}=0.9^{\prime}\right)$ and dense, it appears as a conspicuous object on the DSS image shown in Fig. 3.

\subsection{Other candidates}

Table $2 \mathrm{~d}$ lists further stellar agglomerations reported to the DSH newsgroup between June 2003 and December 2004, which we consider to be possible open clusters from the analytical procedures described in Sect. 2. As in Tables 2a to 2c, positions and angular diameters of the cluster candidates are given, and a DSH designation and the discoverer ID are assigned. It should be noted that 2 of the objects presented in this section (DSH J0629.4+0910 = Alessi 53 and DSH J1959.5+4918 = Patchick 89) turned out to be described by Drake (2005) as objects DC 6 and DC 7, respectively; nevertheless, as in the case of Teutsch 49 (see Sect. 3.2.3), we decided to include these objects as they have been discovered independently.

Although the evidence that the objects listed in Table $2 \mathrm{~d}$ are indeed genuine open clusters is weaker than for the candidates presented in Tables 2a to 2c, their morphologies, CMDs and RDPs are good arguments in favor of their being clusters. However, as with the cluster candidates described in the previous section, deeper studies are necessary to further clarify their nature.

An additional list of 174 stellar fields with evidence that an open cluster might be involved (=Table $2 \mathrm{e}$ ) is available via the CDS. As in Table 2d, the following information is given: DSH ID, discoverer ID, RA J2000, Dec J2000, and the visual diameter $D_{\text {vis }}$.

\section{Conclusions}

An inspection of several Milky Way fields using DSS and 2MASS $J H K_{\mathrm{S}}$ imagery has led to the discovery of 66 stellar aggregates that are thought to be open clusters or, at least, promising open cluster candidates due to their appearance, their stellar density distributions, and their CMDs. For 9 of these objects, we were able to determine preliminary distances, reddenings, and ages by fitting their CMDs with solar metallicity Padova isochrones and found distances $d=1.6-4.6 \mathrm{kpc}$, reddenings $E(B-V)=0.52-2.55$, and ages ranging from less than $10 \mathrm{Myr}$ to $\approx 1$ Gyr. Additionally, distances and reddenings were derived for 10 clusters by using their helium-burning red clump stars as standard candles, with the values between $3.6 \mathrm{kpc}$ and $11.9 \mathrm{kpc}$ found for the distances and between 0.54 and 2.11 for the reddenings. Furthermore, we note the presence of X-ray sources in the immediate vicinity of 2 clusters presented here, Teutsch 42 and Kronberger 81; however, the question whether these are indeed physically connected with the clusters or not cannot be answered from the available data and therefore shall be the object of a deeper investigation.

The high number of newly discovered cluster candidates, as presented in this paper, can be seen as a sign of the low completeness level of even the most up-to-date catalogues. Considering that no systematic all-sky survey for open clusters has been carried out yet on 2MASS or on the Second Palomar Observatory Sky Survey images, it can be assumed that the chances are high that a number of unknown galactic clusters detectable in optical and infrared wavelengths is still awaiting discovery. It should be noted, however, that further studies are required to confirm that the objects presented here are true clusters and to determine their precise fundamental parameters.

\section{Addendum}

During our survey for new open cluster candidates on DSS and 2MASS, we noted a number of galactic nebulae and galaxies that were found not to be listed in any current database. The most impressive of these objects are listed in Table 3, with a more extensive list (Tables $4 \mathrm{a}$ and $4 \mathrm{~b}$ ) available via the CDS. All objects are tentatively classified on the basis of their 
brightness and morphology on DSS-2 blue, red, and infrared images. However, further detailed investigations are needed to establish their true nature.

Acknowledgements. This publication made use of data products from the Two Micron All Sky Survey, which is a joint project of the University of Massachusetts and the Infrared Processing and Analysis Center/California Institute of Technology, funded by the National Aeronautics and Space Administration and the National Science Foundation. Extensive use was also made of the SIMBAD and WEBDA databases, the ESO Online Digitized Sky Survey facility, and the Aladin Interactive Sky Atlas. Furthermore, special thanks go to: A. Block of the NOAO, USA, and D. J. Frew of the Macquarie University, Australia, for providing extra information on some of our planetary nebulae candidates; to W. S. Dias of the University of São Paulo; B. Skiff of Lowell Observatory; O. Brazell of the Webb Society; and A. Moitinho of the Observatorio Astronómico Nacional, México, for reading and commenting on the manuscript; and to M. Sinhuber for extensive technical support. Last but not least, the authors of this study would like to thank all DSH members for their enthusiasm and far-reaching support.

The DSH collaboration is a group of amateur and professional astronomers founded in June 2003. Its intentions are the discovery of deep sky objects not yet published in professional literature and the improvement of observational data for already known objects.

\section{References}

Abt, H. A., \& Corbally, C. J. 2000, ApJ, 541, 841

Acker, A., Ochsenbein, F., Stenholm, B., et al. 1992, Strasbourg-ESO Catalogue of Galactic Planetary Nebulae (Strasbourg: CDS)

Alessi, B. S., Moitinho, A., \& Dias, W. S. 2003, A\&A, 410, 565

Alter, G., Balázs, B., \& Ruprecht, J. 1970, Catalogue of Star Clusters and Associations, 2nd edn. (Budapest: Akademia Kiadó)

Alves, D. 2000, ApJ, 539, 732

Ambartsumian, V. A. 1954, Contrib. Byurakan Obs., 15, 3

Archinal, B. A., \& Hynes, S. J. 2003, Star Clusters (Richmond: Willmann-Bell)

Bica, E., Dutra, C. M., \& Barbuy, B. 2003a, A\&A, 397, 177

Bica, E., Dutra, C. M., Soares, J. B., \& Barbuy, B. 2003b, A\&A, 404, 223

Bica, E., Bonatto, C., \& Dutra, C. M. 2003c, A\&A, 405, 991

Bica, E., Bonatto, C., \& Dutra, C. M. 2004, A\&A, 422, 555

Block, A. 2005, private communication

Borissova, J., Pessev, P., Ivanov, V. D., et al. 2003, A\&A, 411, 83

Brodskaya, E. S., \& Shajn, P. F. 1958, IzKry, 20, 299B

Buscombe, W. 1998, 13th General Catalogue of MK Spectral Classification (Strasbourg: CDS)

Buscombe, W. 1999, 14th General Catalogue of MK Spectral Classification (Strasbourg: CDS)

Cutri, R. M., et al. 2003, The 2MASS Catalogue of Point Sources, University of Massachusetts and Infrared Processing and Analysis Center (IPAC)/ California Institute of Technology

Dias, W. S., Alessi, B. S., Moitinho, A., \& Lépine, J. R. D. 2002, A\&A, 389, 871; online version available via http://www. astro.iag.usp.br/ wilton

Drake, A. J. 2005, A\&A, 435, 545

Drommanget, J., \& Nys, O. 2002, Catalog of Components of Double and Multiple stars (Strasbourg: CDS)

Dutra, C. M., \& Bica, E. 2000, A\&A, 359, L9
Dutra, C. M., Santiago, B. X., \& Bica, E. 2002, A\&A, 381, 219

Dutra, C. M., Bica, E., Soares, J. B., \& Barbuy, B. 2003, A\&A, 400, 533

ESA 1997, The Hipparcos and TYCHO Catalogues (European Space Agency ESA)

Frew, D. J. 2005a, private communication

Frew, D. J. 2005b, private communication

Frew, D. J. 2005c, private communication

Frew, D. J. 2005d, private communication

Girardi, L., \& Salaris, M. 2001, MNRAS, 323, 109

Girardi, L., Bartelli, G., Bressan, A., et al. 2002, A\&A, 391, 195

Grocholski, A. J., \& Sarajedini, A. 2002, AJ, 123, 1603

Harris, D. E., et al. 1994, EINSTEIN Observatory catalog of IPC $\mathrm{X}$-ray sources (Strasbourg: CDS)

Høg, E., Fabricius, C., Makarov, V. V., et al. 2000, The Tycho-2 Catalogue of the 2.5 Million Brightest Stars, A\&A, 355, L27

Ivanov, V. D., Borissova, J., Pessev, P., Ivanov, G. R., \& Kurtev, R. 2002, A\&A, 394, 1

Kharchenko, N., \& Schilbach, E. 1995, Astron. Nachr., 316, 91

Minkowski, R. 1946, PASP, 58, 305

Kuchar, T. A., \& Clark, F. O. 1997, ApJ, 488, 224

Landolt-Börnstein 1996, NS., Vol. VI/3b, 15, 18

Lanning, H. H., \& Meakes, M. 2000, PASP, 112, 251

Lauberts, A. 1982, The ESO/Uppsala Survey of the ESO (B) Atlas, European Southern Observatory, Garching bei München

Lynds, B. T. 1962, ApJS, 7, 1

Lynds, B. T. 1965, ApJS, 12, 163

Lynga, G. 1987, Computer Based Catalogue of Open Cluster Data, 5th ed. (Strasbourg: CDS)

Magakian, T. Y. 2003, A\&A, 399, 141

Minkowski, R. 1946, Publ. Astron. Soc. Pac., 58, 305

Neckel, T., \& Vehrenberg, H. 1985, Atlas galaktischer Nebel I. RA 0h00m-12h00m, Dekl. +90--33 deg, Duesseldorf, Treugesellverlag, 1985

Pfleiderer, J., Weinberger, R., \& Mross, R. 1977, Star Cluster Symposium, Budapest, 39

Reed, B. C. 1998, AJ Suppl. Ser., 115, 271

Rieke, G. H., \& Lebofsky, M. J. 1985, ApJ, 288, 618

Roman, A. T., Iwata, I., \& Saito, M. 2000, ApJS, 127, 27

ROSAT Consortium 2000, The Second ROSAT Source Catalog of Pointed Observations (Strasbourg: CDS)

Sanders, W. L. 1971, A\&A, 14, 226

Saurer, W., Seeberger, R., Weinberger, R., \& Ziener, R. 1994, AJ, 107, 2101

Setteducati, A. F., \& Weaver, M. F. 1962, Newly Found Stellar Clusters, University of California, Berkeley

Sharpless, S. 1959, ApJS, 4, 257

Skiff, B. 2005, private communication

Skrutskie, M., et al. 1997, in The Impact of Large Scale Near-IR Sky Surveys, ed. Garzon et al. (Netherlands: Kluwar), 210, 187

Töpler, R. 1998, in Interstellarum 13, VdS - Fachgruppe Deep-SkyBeobachtung, Eigenverlag, 7

Urban, S. E., Corbin, T. E., Wycoff, G. L., et al. 2001, AAS, 33, 1494

Van Genderen, A. M. 2001, A\&A, 366, 508

Voges, W., Aschenbach, B., Boller, T., et al. 1999, A\&A, 349, 389

Wright, C. O., Egan, M. P., Kraemer, K. E., et al. 2003, AJ, 125, 359

Worley, C. E., \& Douglass, G. G. 1997, The Washington Visual Double Star Catalogue, 1996.0, A\&AS, 125, 523

Zacharias, N., et al. 2003, AJ, 127, 3043 


\section{Online Material}


M. Kronberger et al.: New galactic open cluster candidates, Online Material p 2
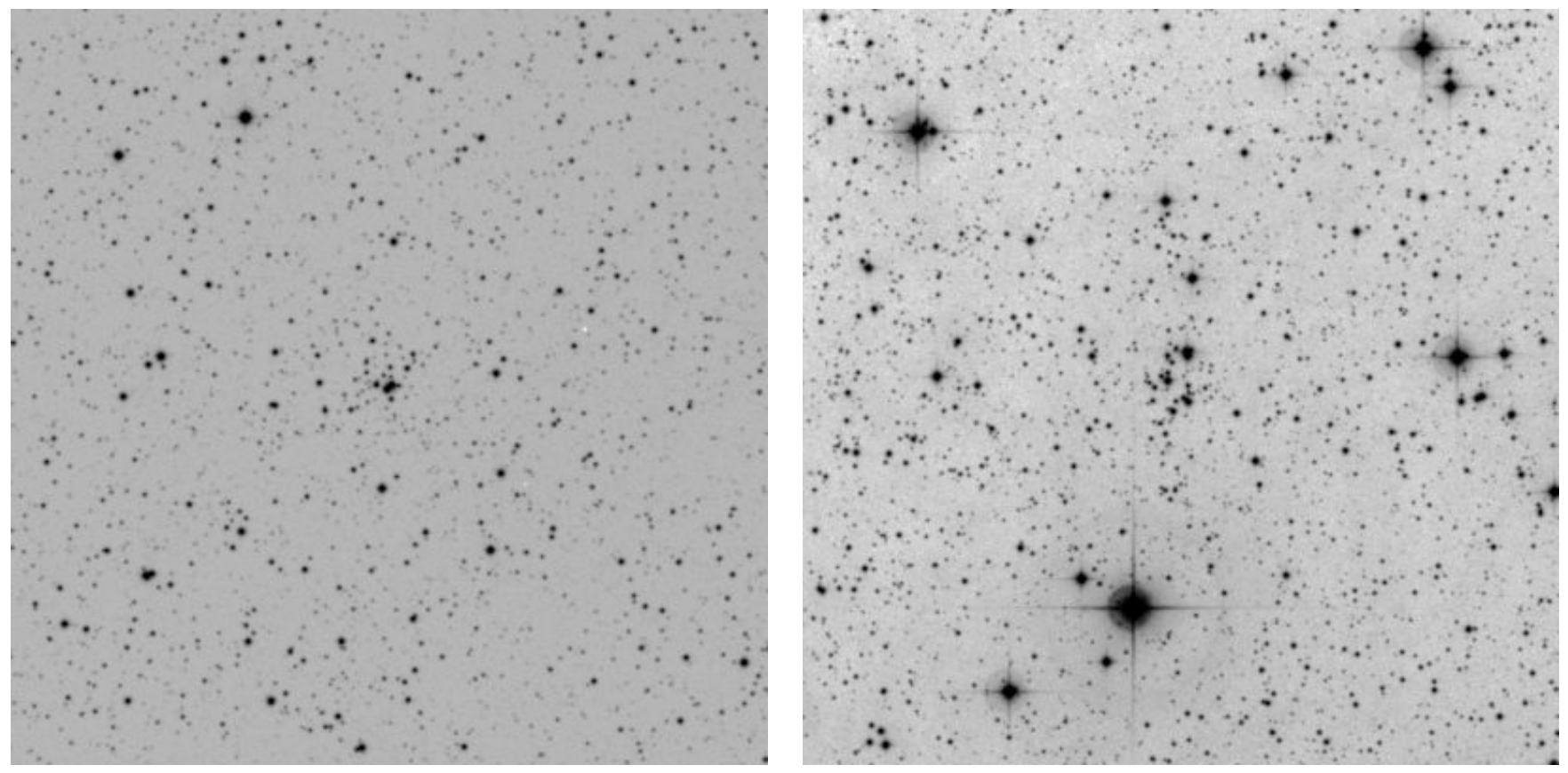

Juchert 9

\section{Kronberger 1}

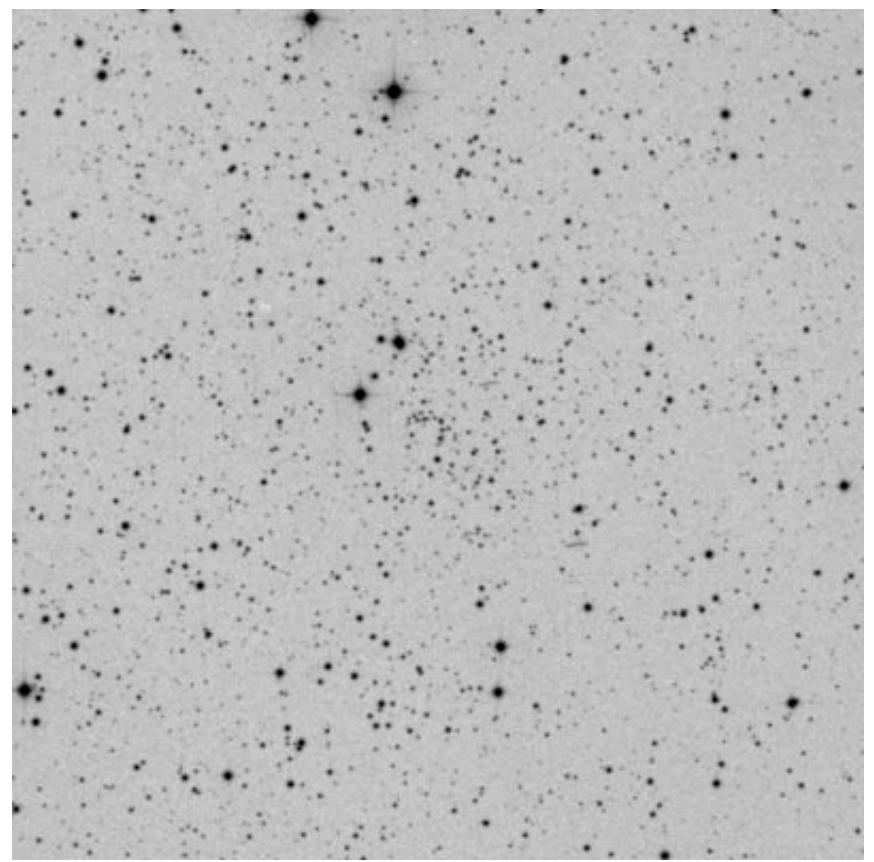

Teutsch 51

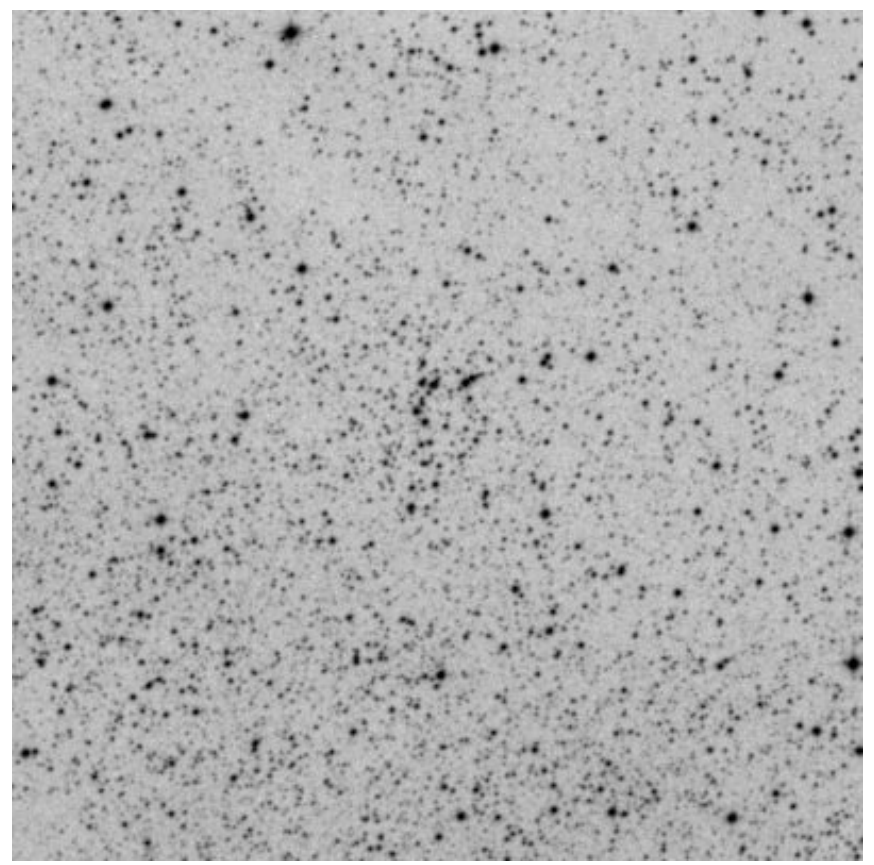

Teutsch 80

Fig. 1. Second generation DSS red images of the cluster candidates presented in Table 2 a. The field of view is in each case $8^{\prime} \times 8^{\prime}$. North is up and East is left. 
M. Kronberger et al.: New galactic open cluster candidates, Online Material p 3

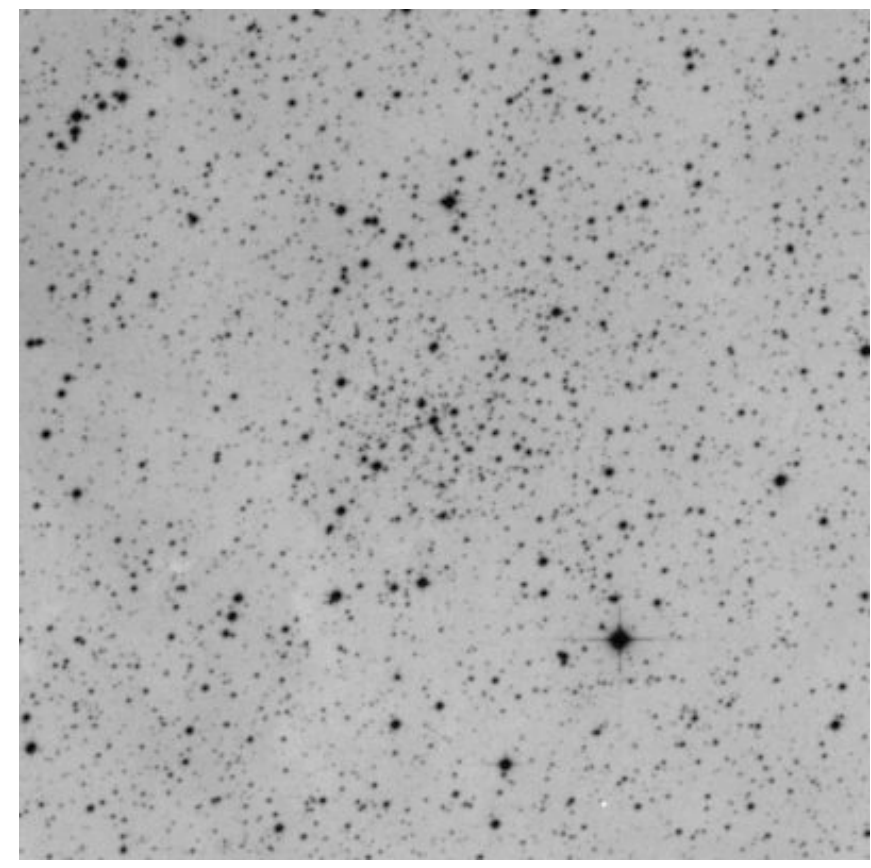

Teutsch 84

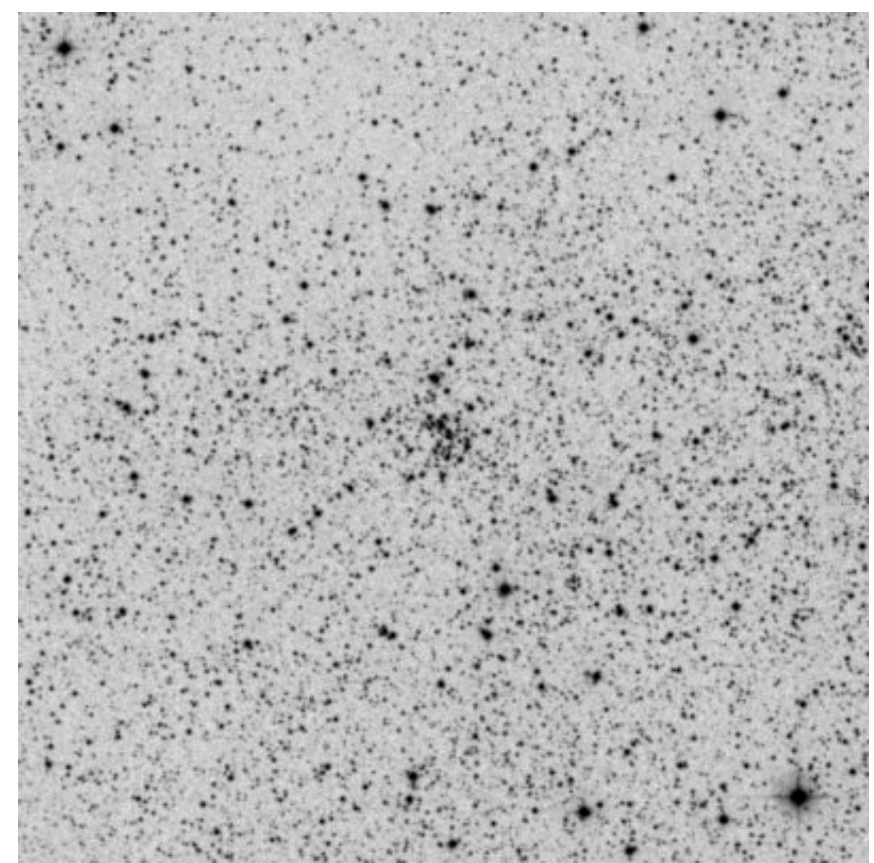

Kronberger 79

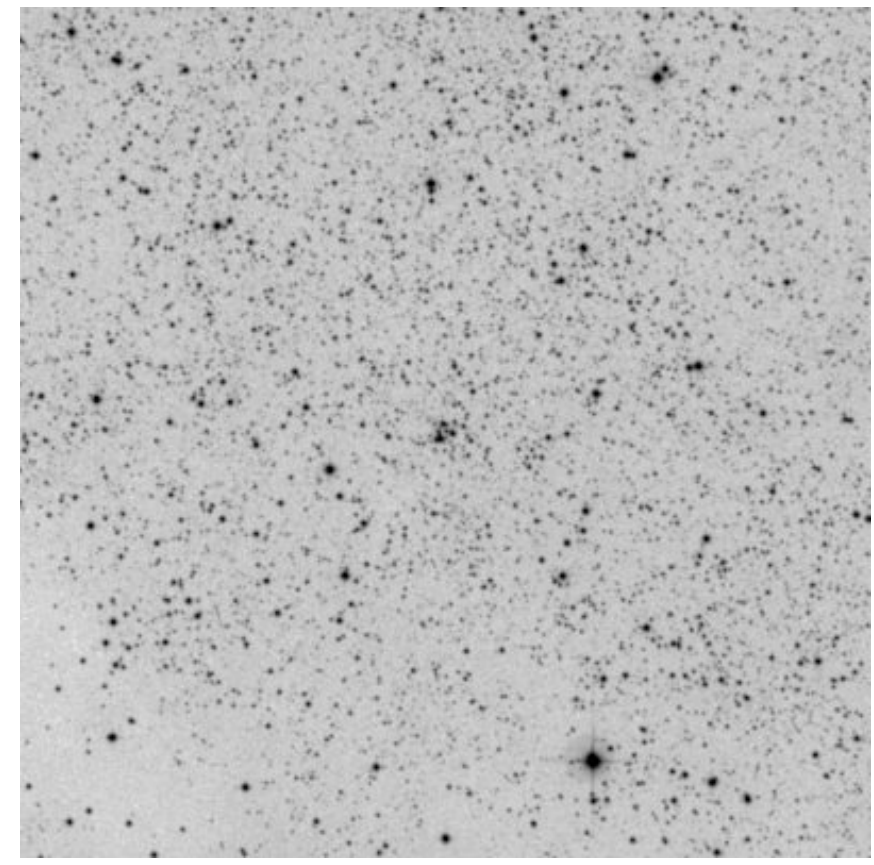

Teutsch 42

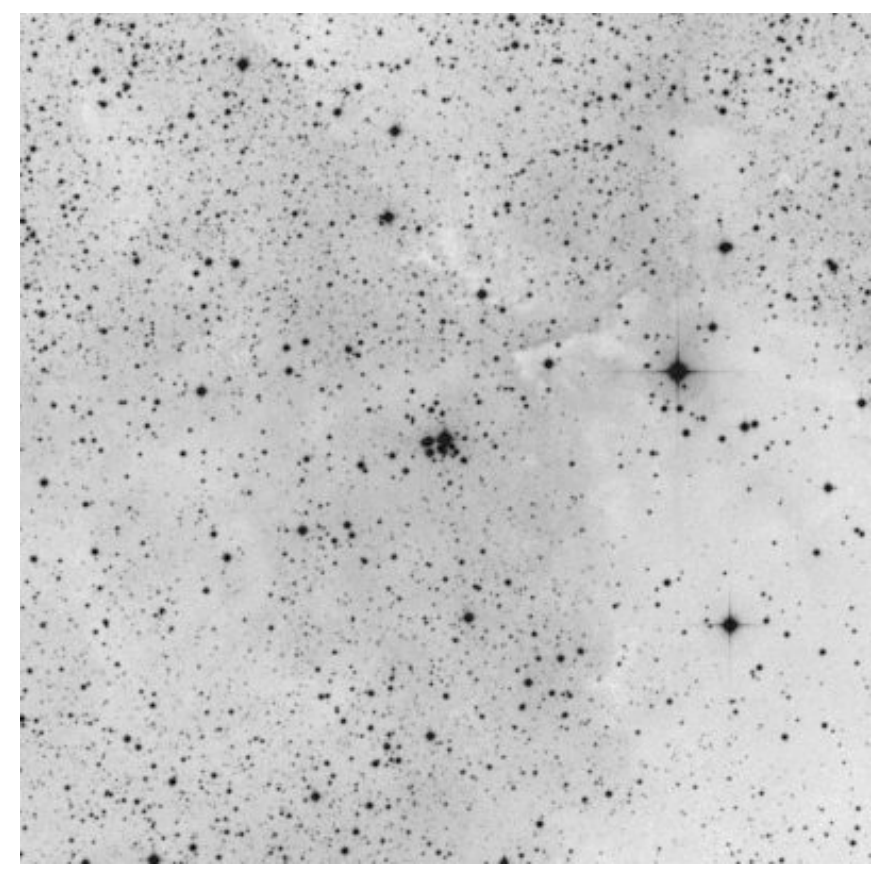

ADS 13292

Fig. 1. continued. 
M. Kronberger et al.: New galactic open cluster candidates, Online Material p 4

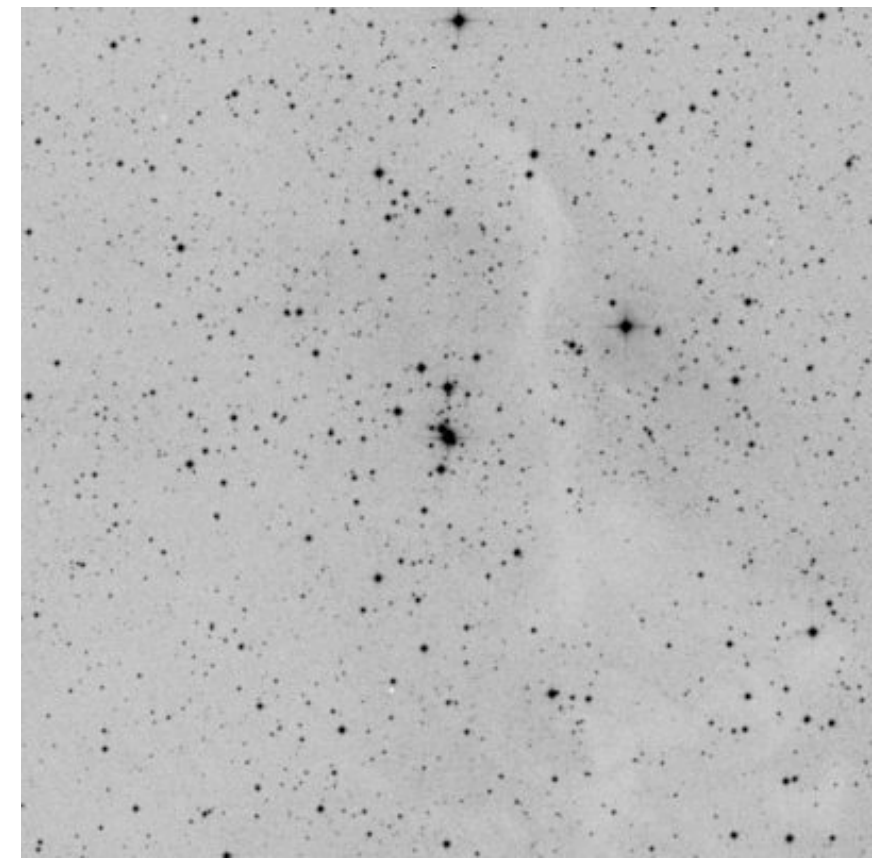

Teutsch 30

Fig. 1. continued. 
M. Kronberger et al.: New galactic open cluster candidates, Online Material p 5

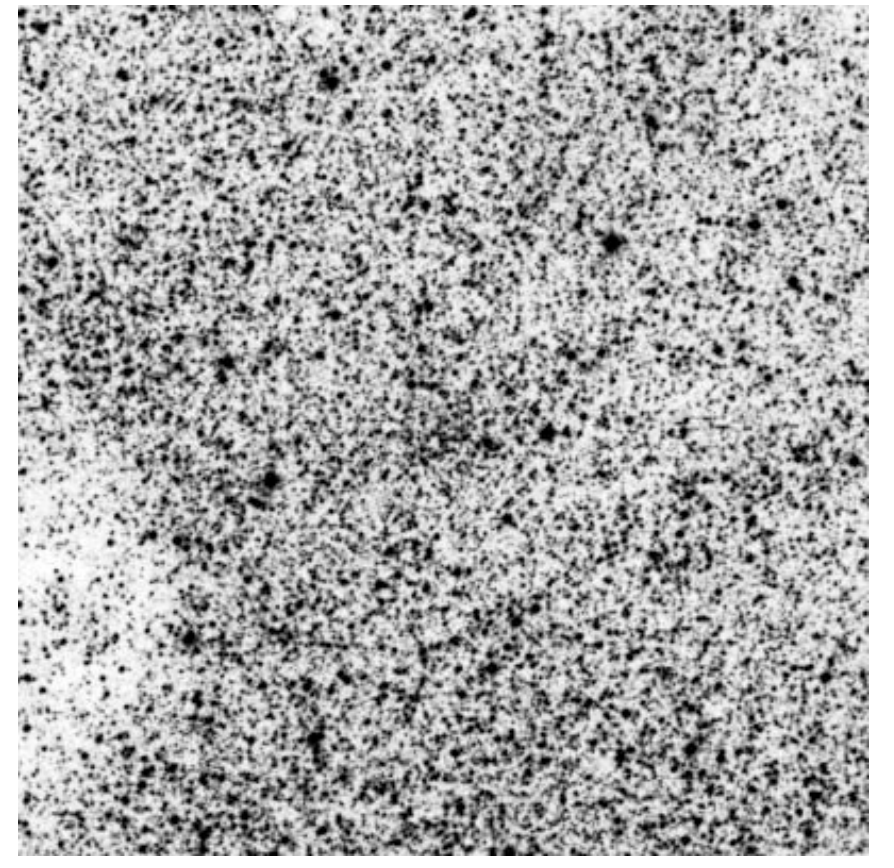

Teutsch 79

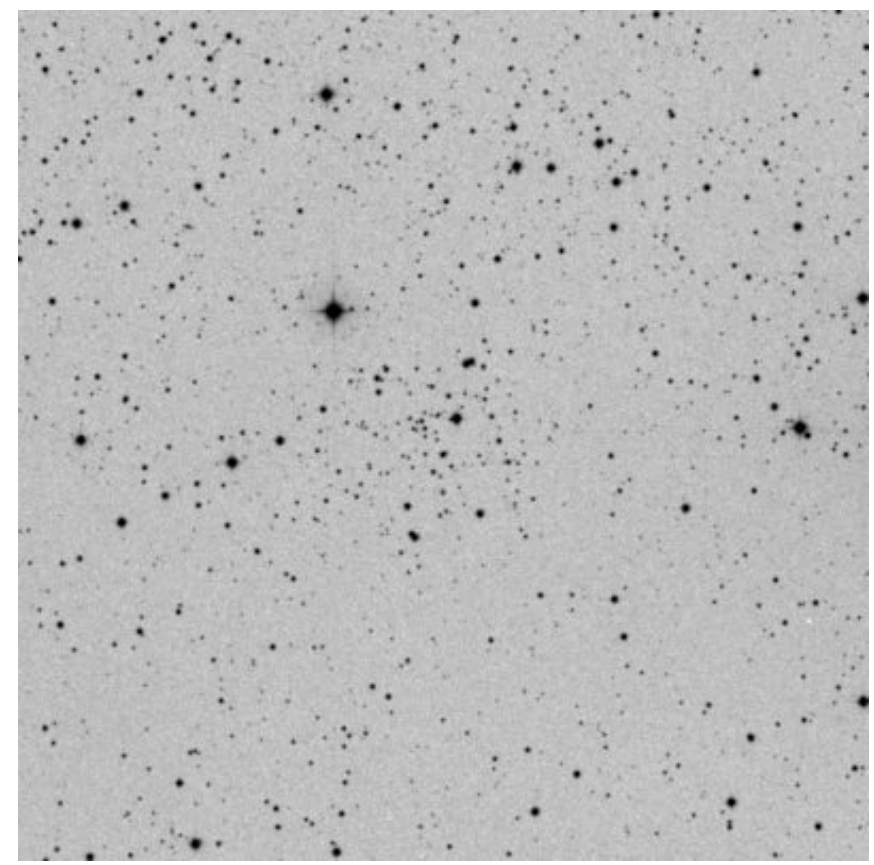

Juchert 11

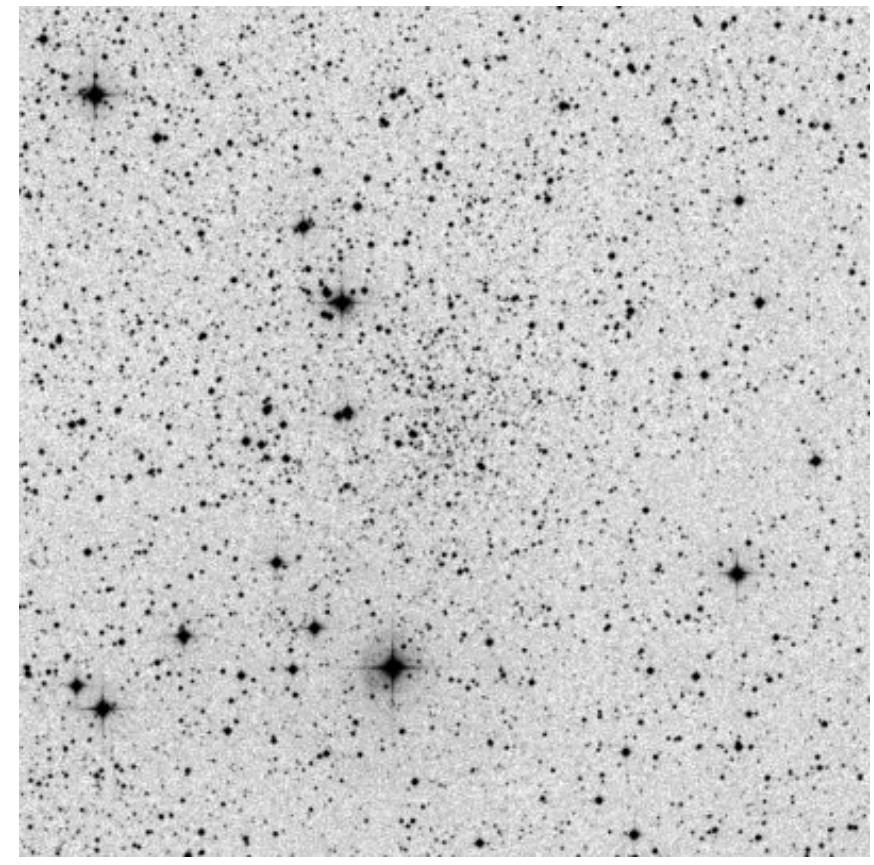

Kronberger 81

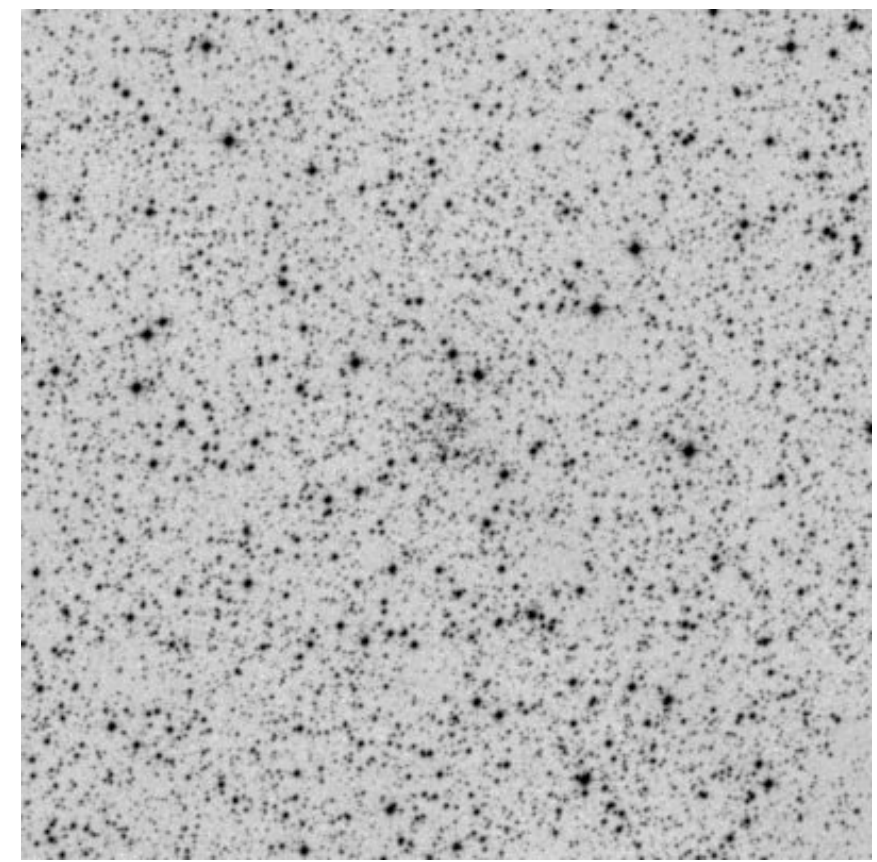

Teutsch 48

Fig. 2. Second generation DSS red images of the cluster candidates presented in Table $2 \mathrm{~b}$. The field of view is in each case $8^{\prime} \times 8^{\prime}$. North is up and East is left. 
M. Kronberger et al.: New galactic open cluster candidates, Online Material p 6

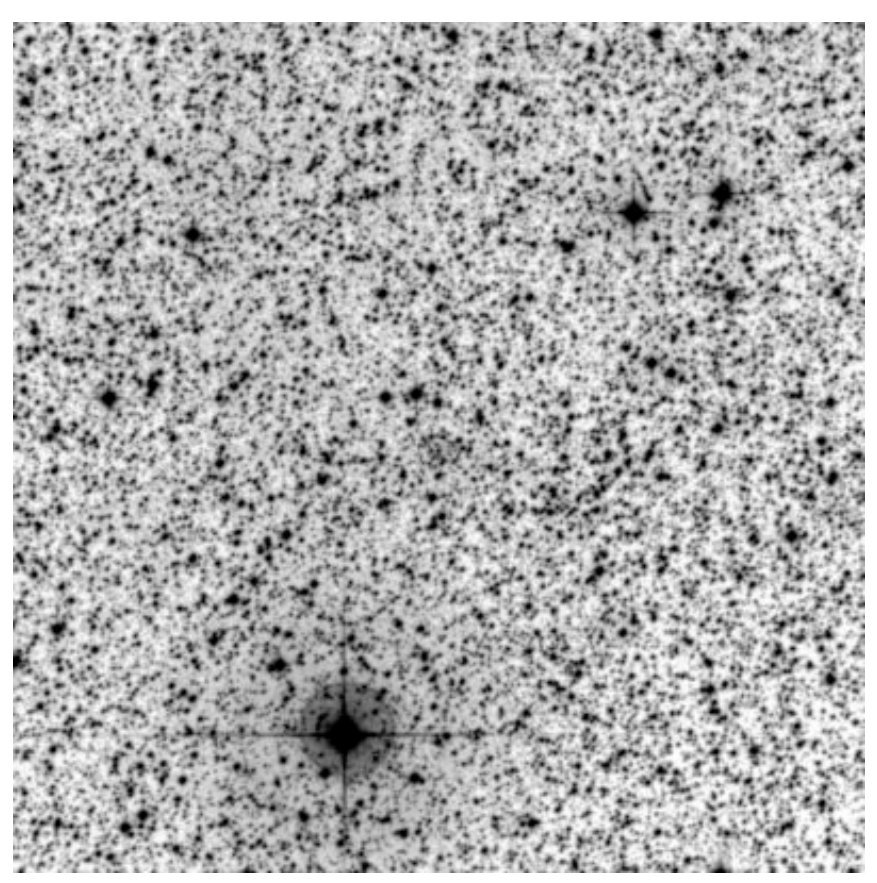

Kronberger 39

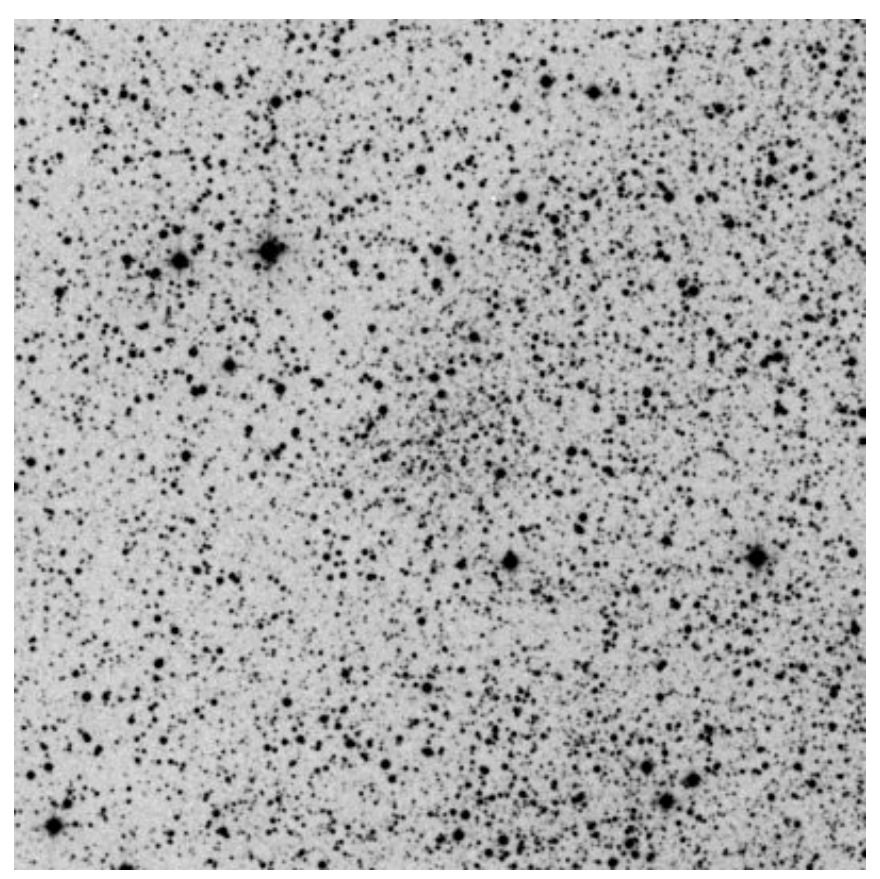

Alessi 57

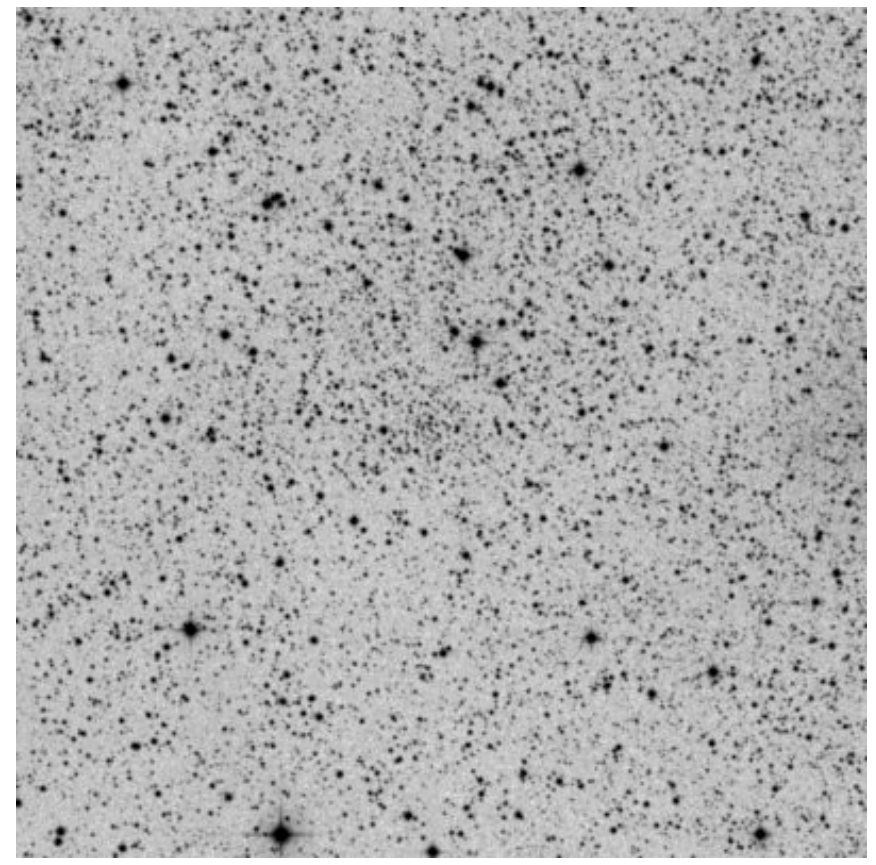

Alessi 56

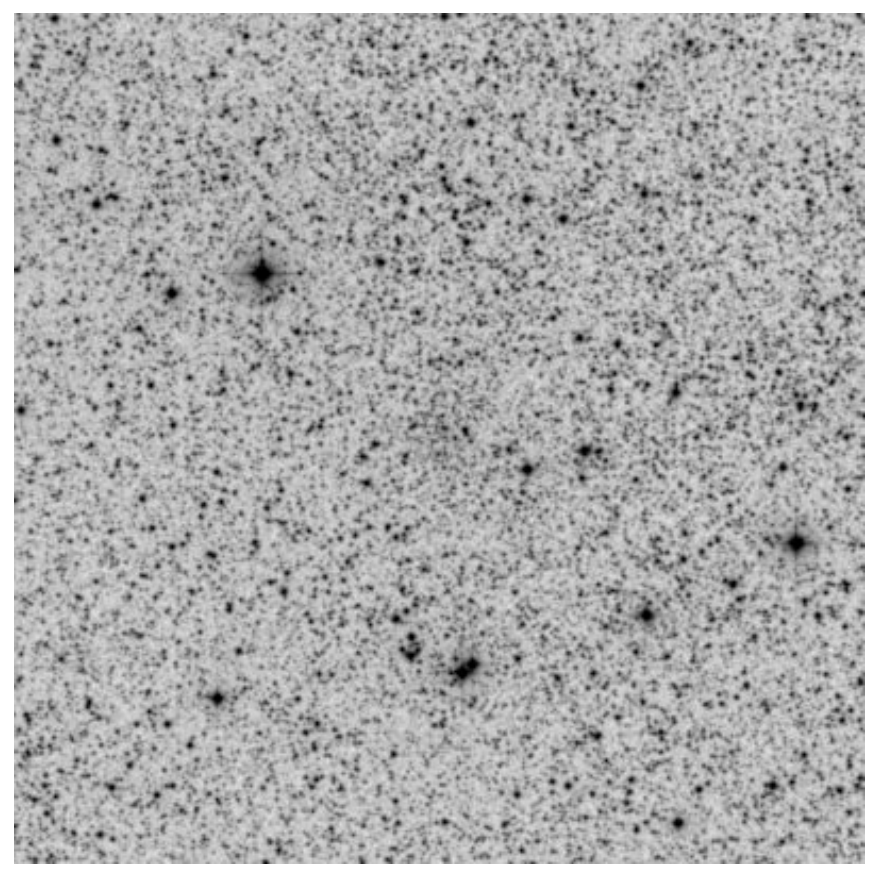

Kronberger 31

Fig. 2. continued. 
M. Kronberger et al.: New galactic open cluster candidates, Online Material p 7

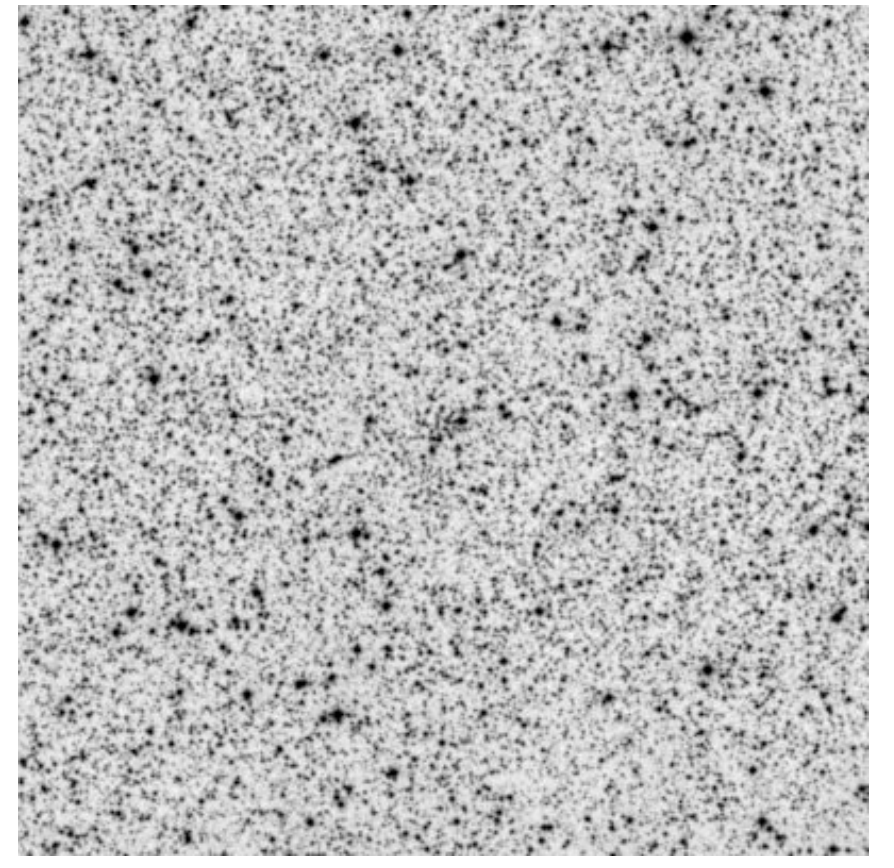

Teutsch 43

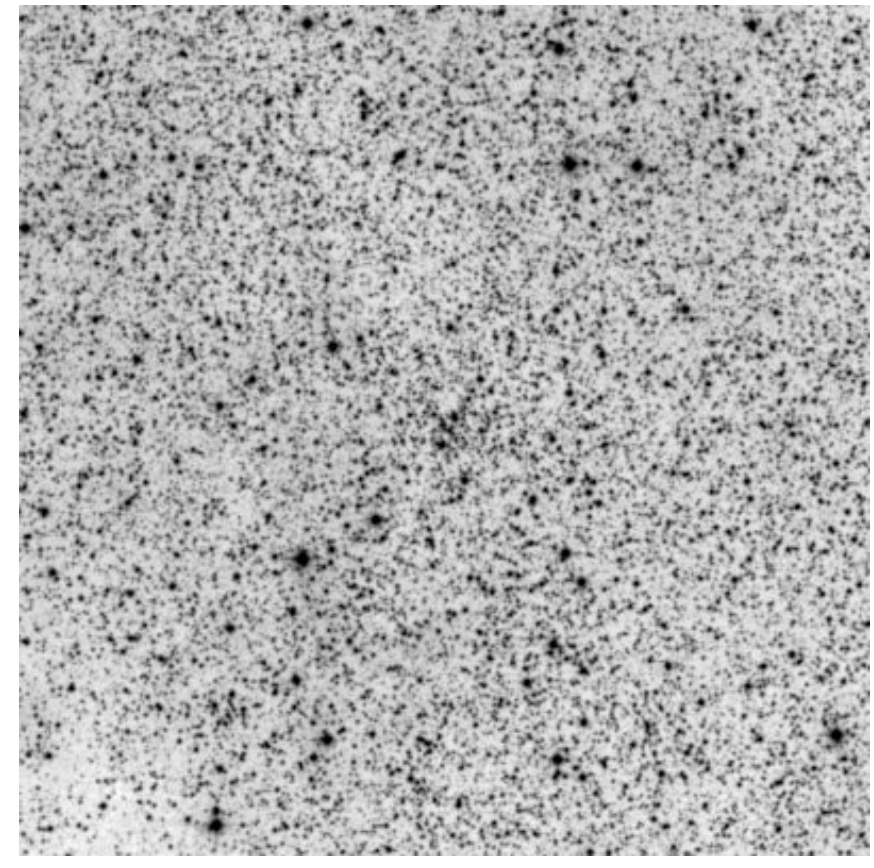

Kronberger 4

Fig. 2. continued. 
M. Kronberger et al.: New galactic open cluster candidates, Online Material p 8

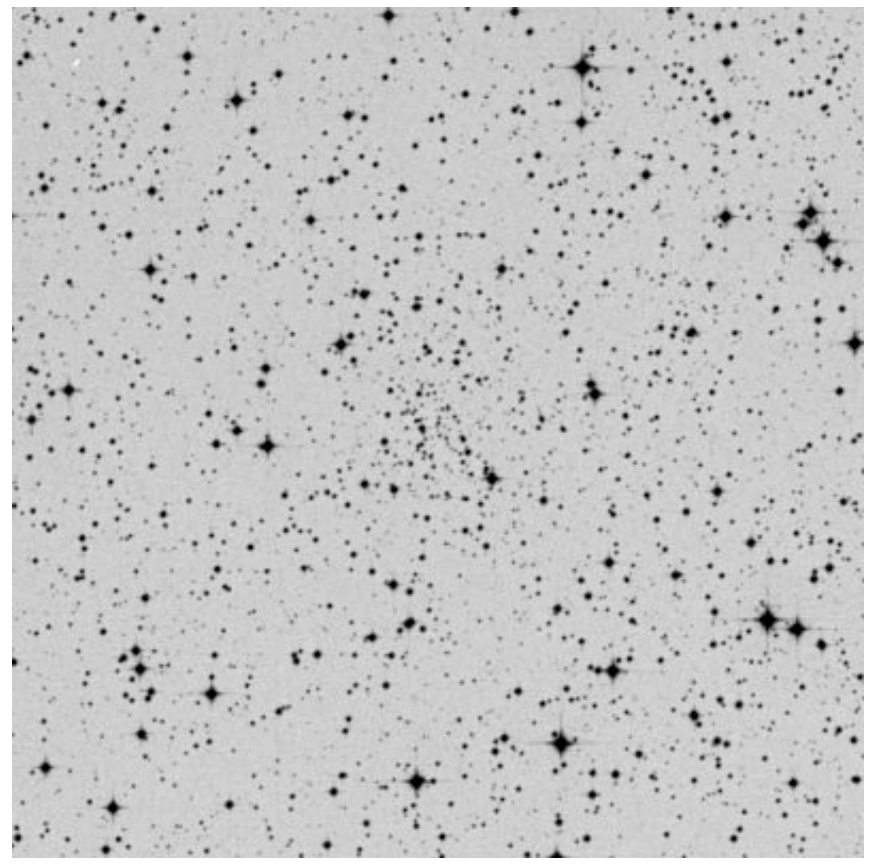

Teutsch 49

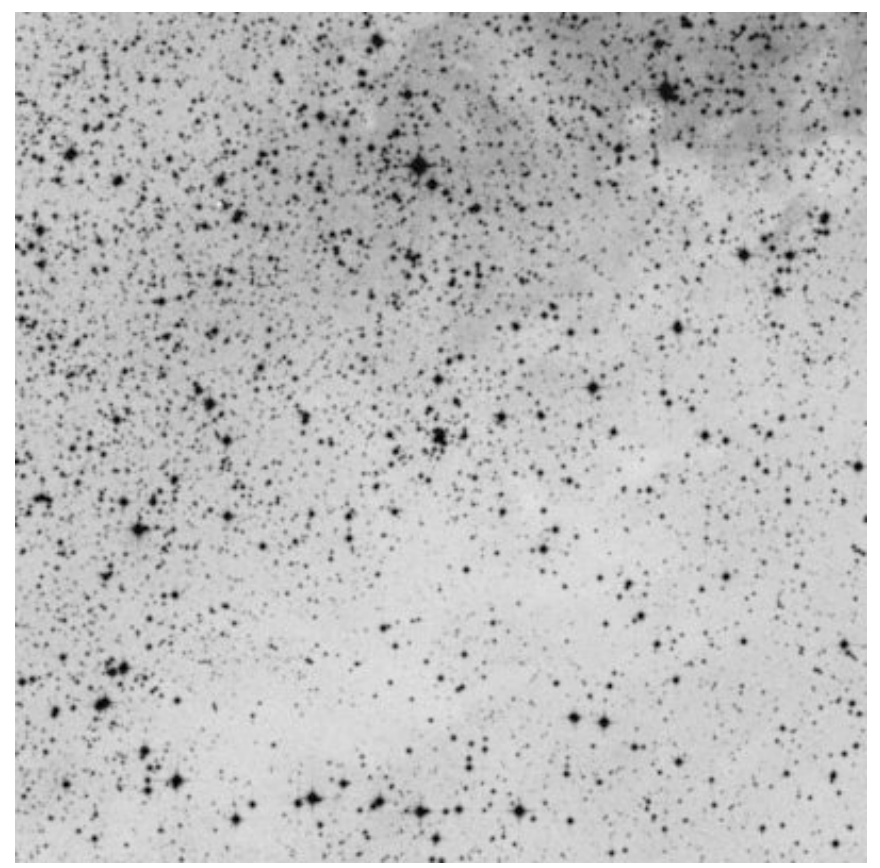

Kronberger 25

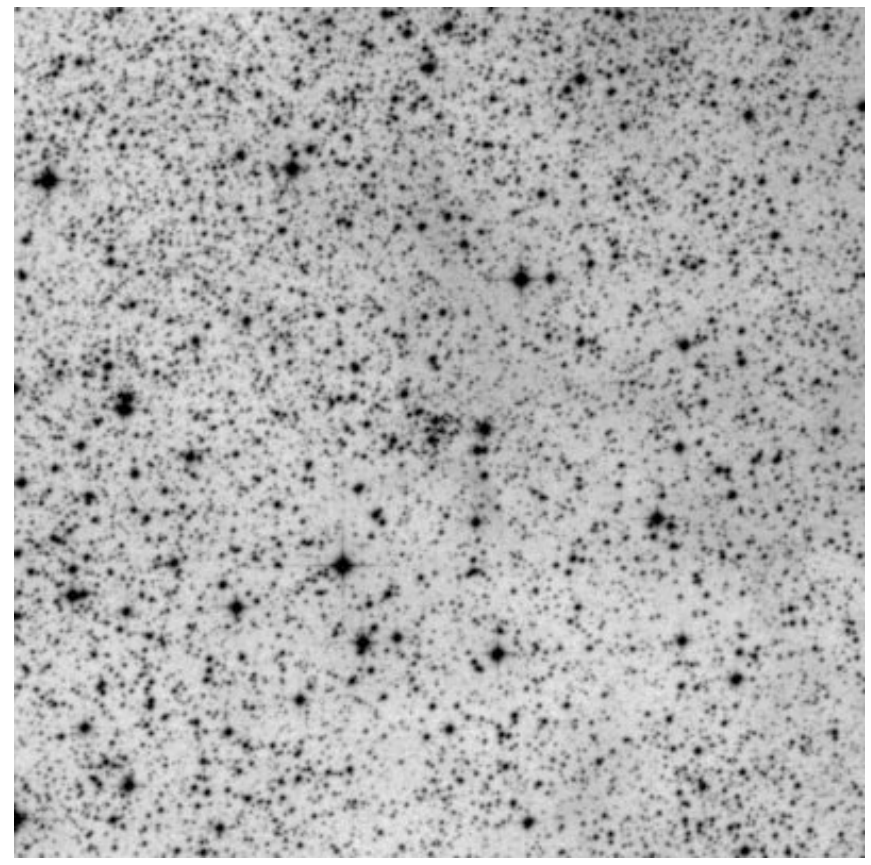

Teutsch 31

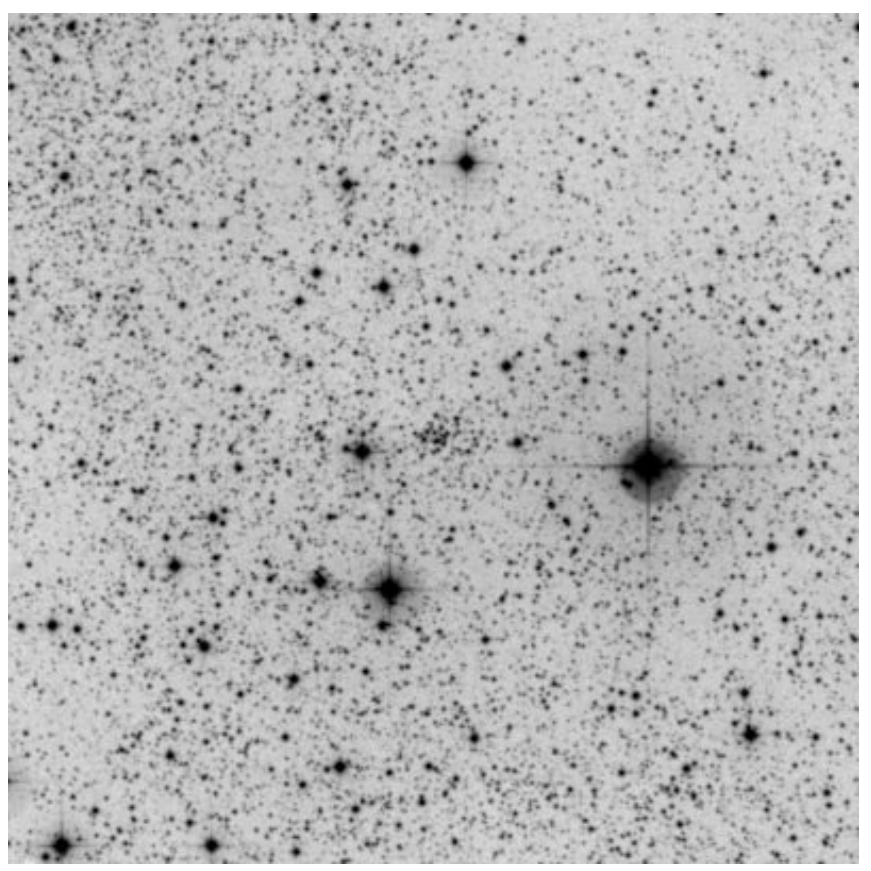

Kronberger 54

Fig. 3. Second generation DSS red images of the cluster candidates presented in Table 2c. The field of view is in each case $8^{\prime} \times 8^{\prime}$. North is up and East is left. 
M. Kronberger et al.: New galactic open cluster candidates, Online Material p 9

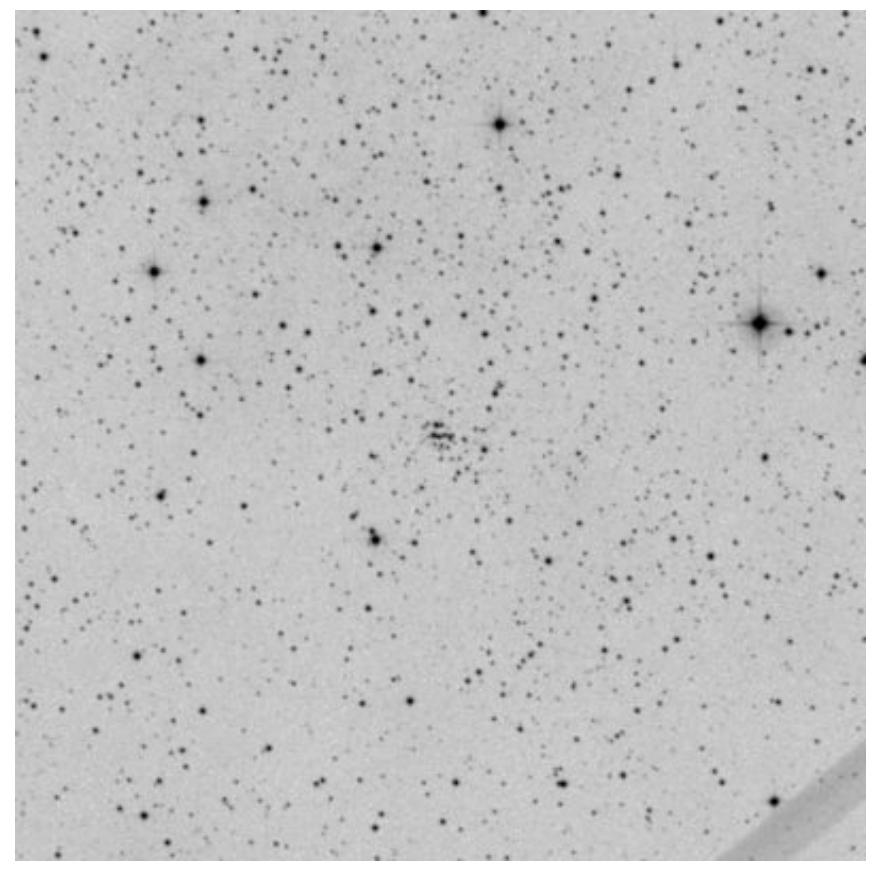

Kronberger 80

Fig. 3. continued. 
M. Kronberger et al.: New galactic open cluster candidates, Online Material p 10
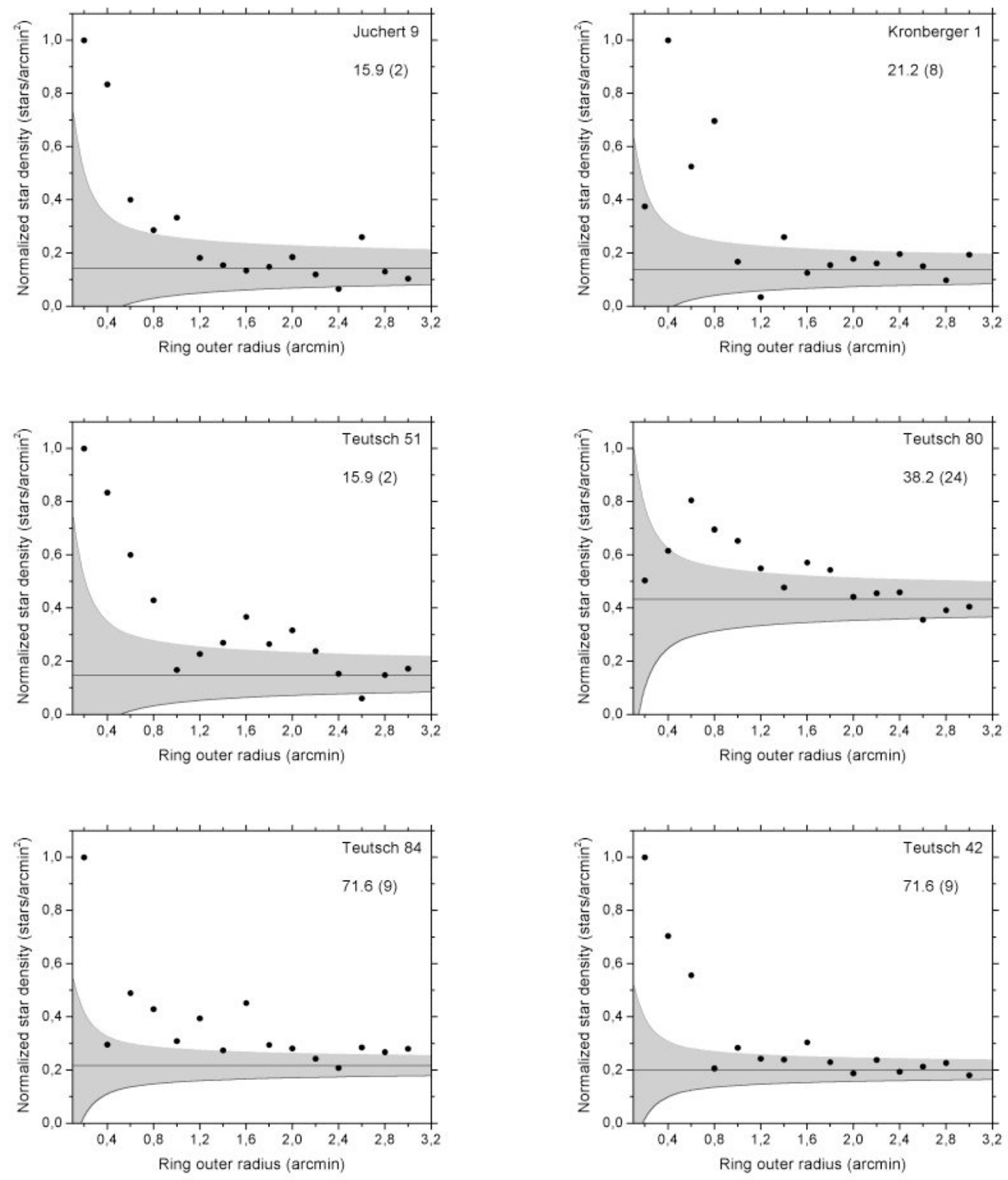

Fig. 4. RDPs of the cluster candidates presented in Table 2a, with the vertical scale normalized to the maximum stellar density in the cluster field. In each diagram, the maximum stellar density (in stars $/ \operatorname{arcmin}^{2}$ ) and, in brackets, the actual number of stars observed in this area are given. The mean density of the background is shown in each diagram as a horizontal line, with the $2 \sigma$ error ranges of the background indicated as shaded areas. Only 2MASS sources with $H<15.5$ were taken into account. 
M. Kronberger et al.: New galactic open cluster candidates, Online Material p 11
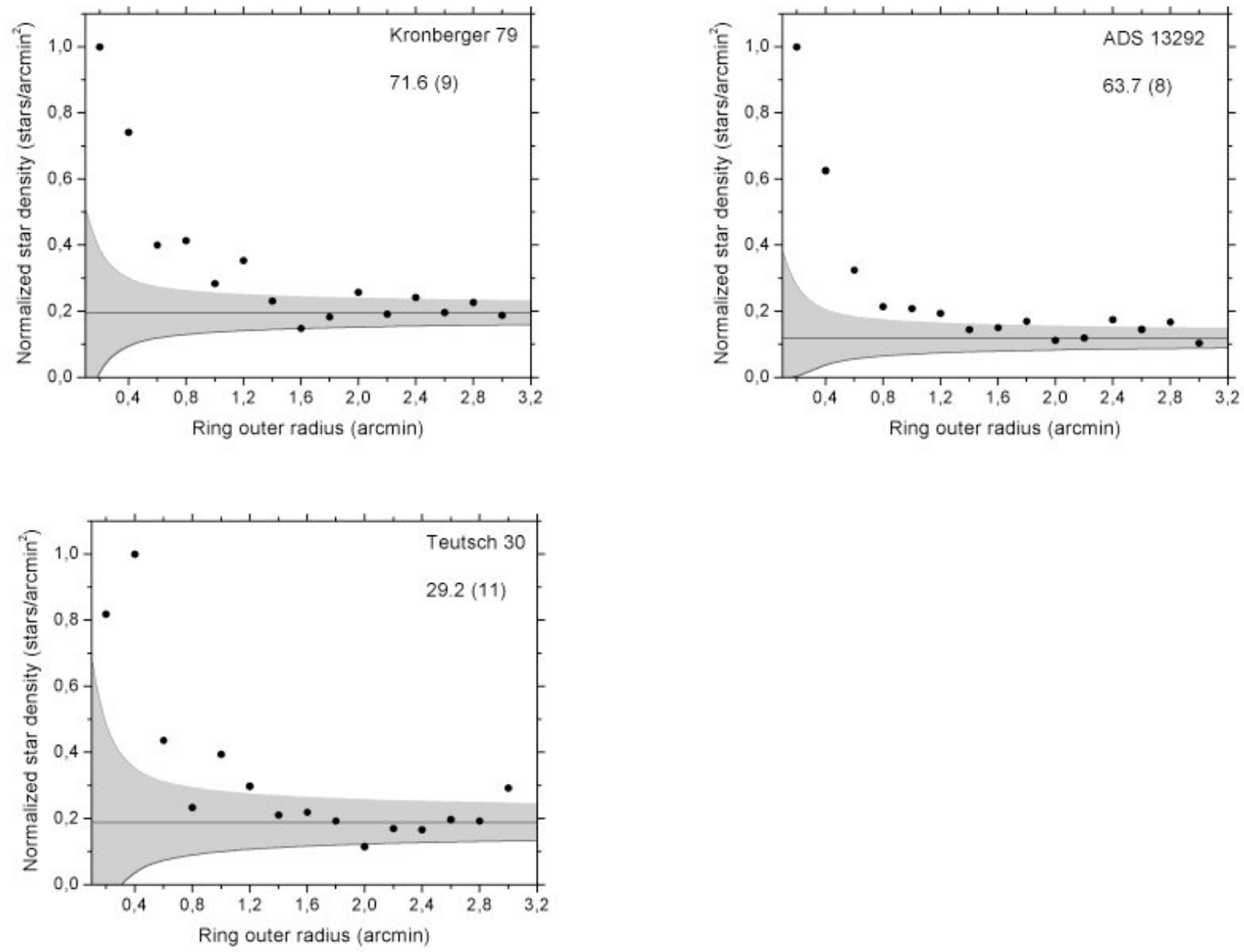

Fig. 4. continued. 
M. Kronberger et al.: New galactic open cluster candidates, Online Material p 12
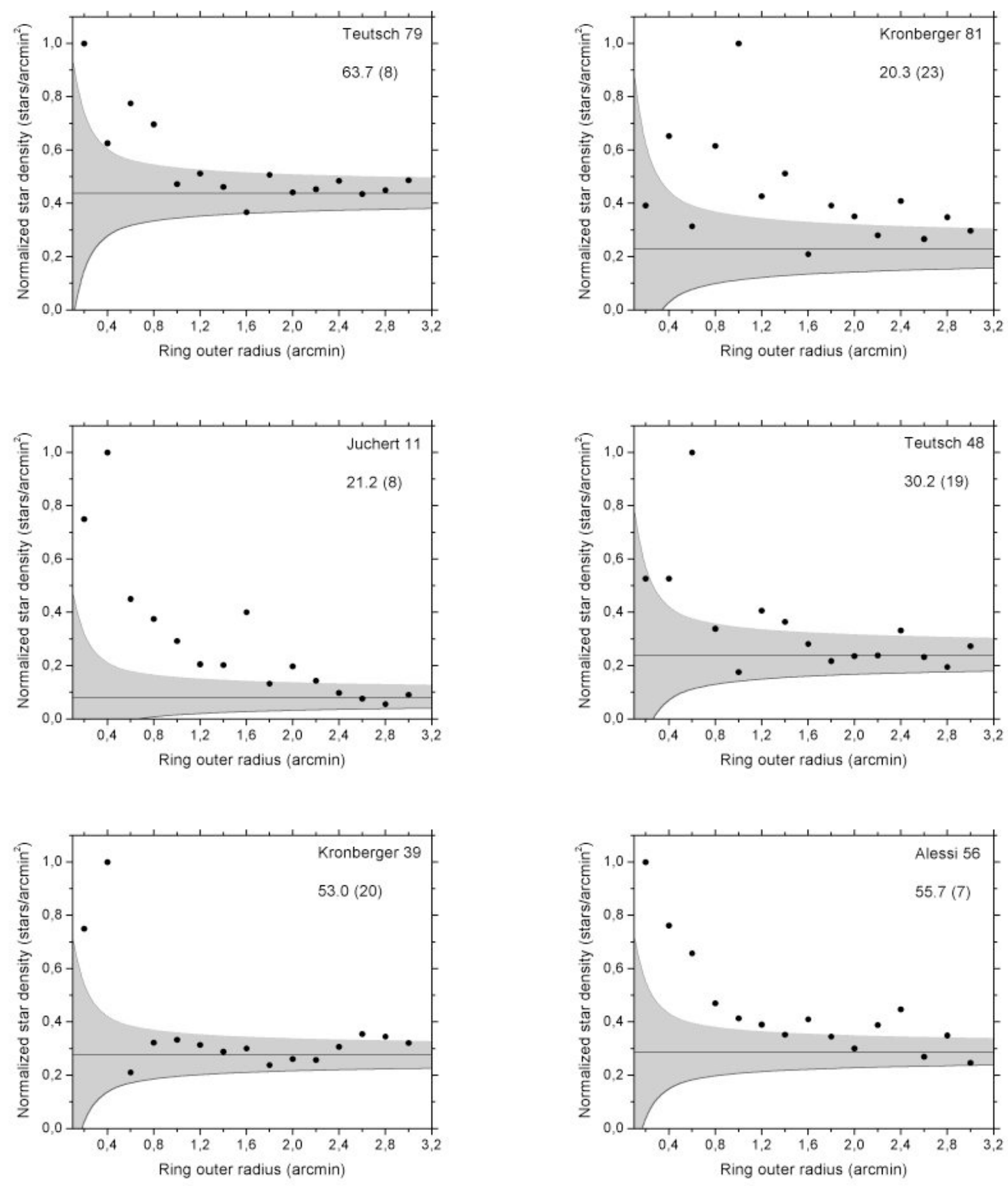

Fig. 5. RDPs of the cluster candidates presented in Table $2 b$, with the vertical scale normalized to the maximum stellar density in the cluster field. In each diagram, the maximum stellar density (in stars $/ \operatorname{arcmin}^{2}$ ) and, in brackets, the actual number of stars observed in this area are given. The mean density of the background is shown in each diagram as a horizontal line, with the $2 \sigma$ error ranges of the background indicated as shaded areas. Only 2MASS sources with $H<15.5$ were taken into account. 
M. Kronberger et al.: New galactic open cluster candidates, Online Material p 13
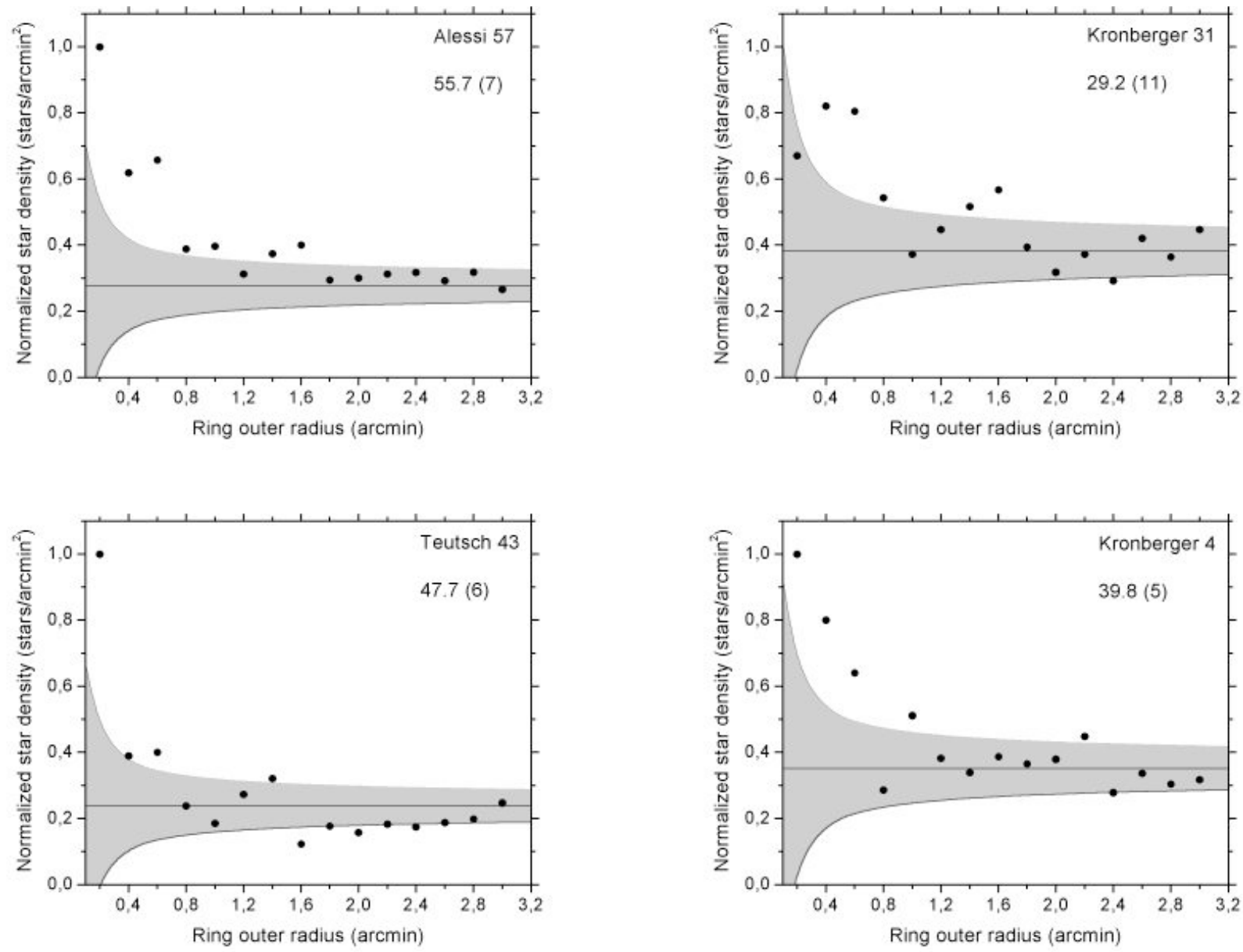

Fig. 5. continued. 
M. Kronberger et al.: New galactic open cluster candidates, Online Material p 14
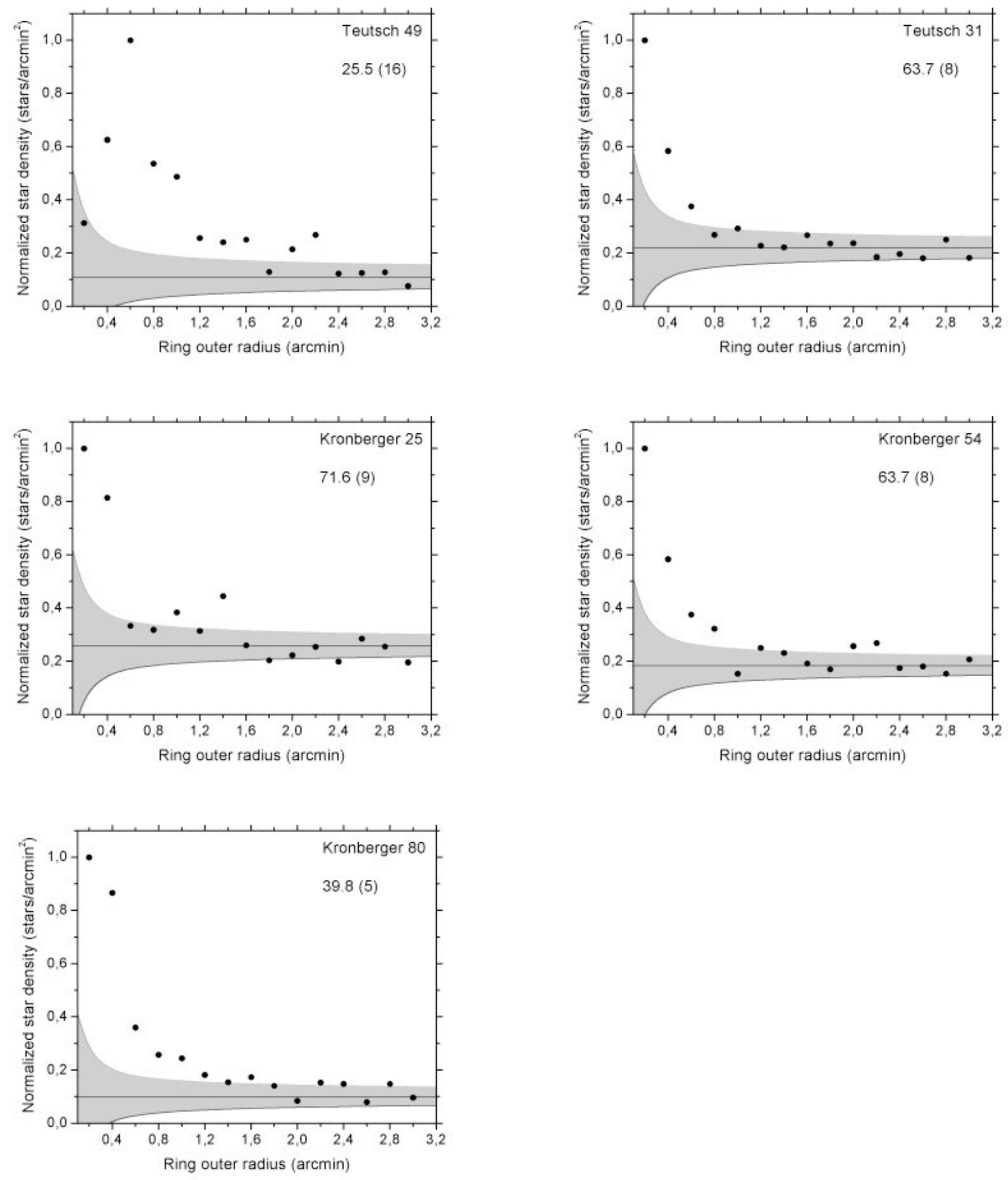

Fig. 6. RDPs of the cluster candidates presented in Table 2c, with the vertical scale normalized to the maximum stellar density in the cluster field. In each diagram, the maximum stellar density (in stars $/ \operatorname{arcmin}^{2}$ ) and, in brackets, the actual number of stars observed in this area are given. The mean density of the background is shown in each diagram as a horizontal line, with the $2 \sigma$ error ranges of the background indicated as shaded areas. Only 2MASS sources with $H<15.5$ were taken into account. 
M. Kronberger et al.: New galactic open cluster candidates, Online Material p 15
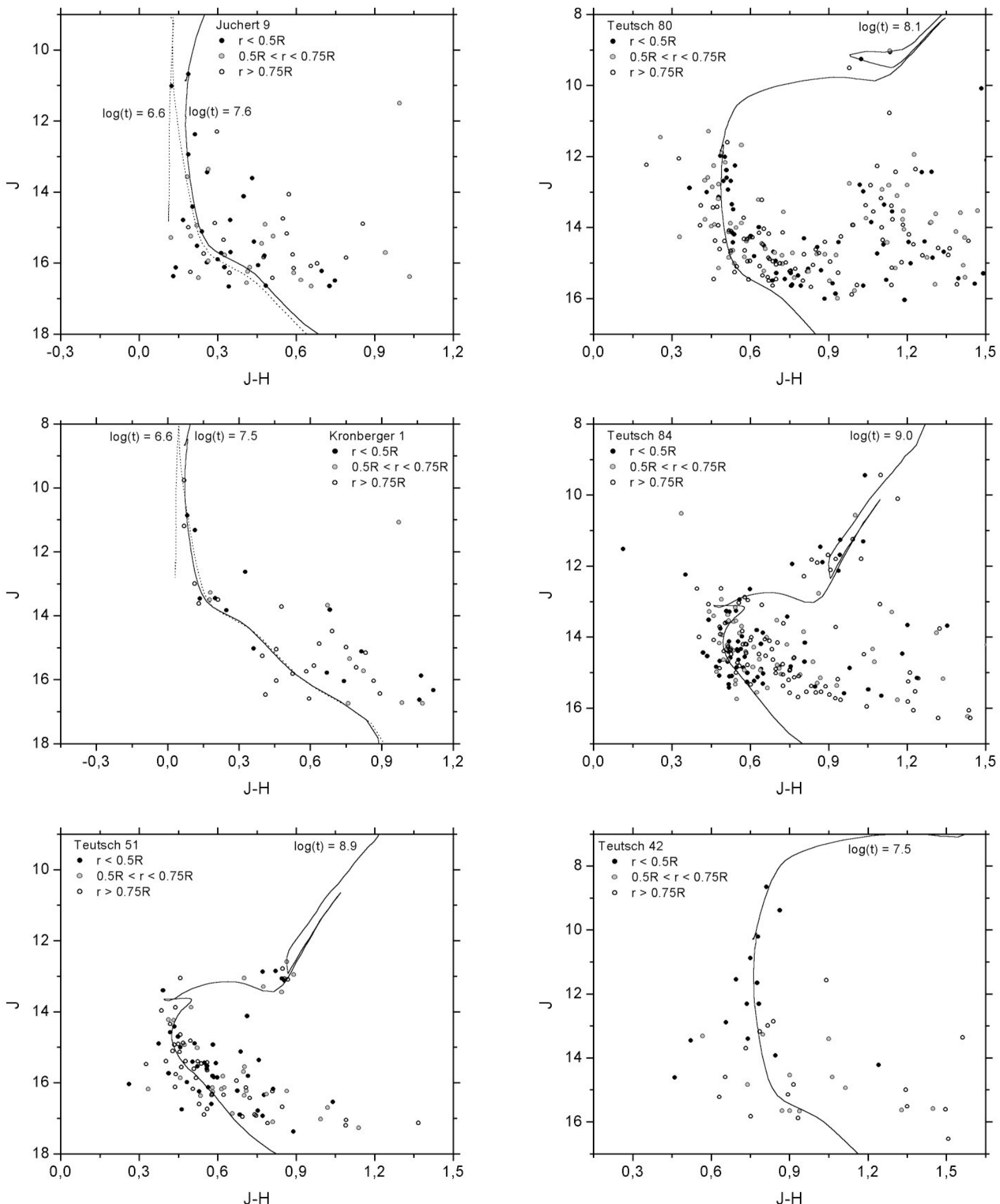

Fig. 7. CMDs of the cluster candidates presented in Table 2a. Each diagram contains only those stars with a distance from the cluster center that is less than the visual cluster radius $R$ and with $J$ and $H$ magnitudes derived either via aperture photometry $\left(r d_{-} f l g=1\right)$ or via point-spread function fitting $\left(r d \_f l g=2\right)$. Also included are the bestfit solar metallicity isochrones of the CMD.

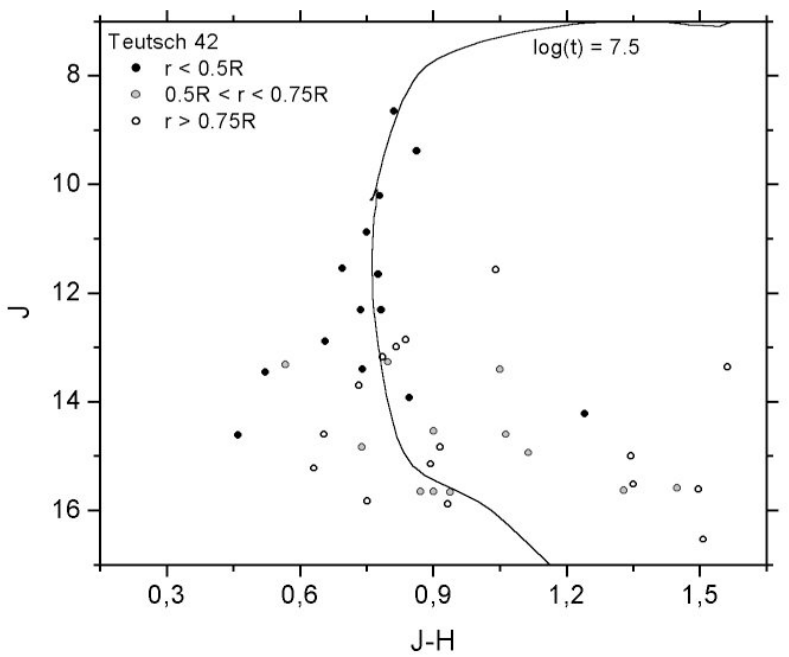

Fig. 7. continued. 
M. Kronberger et al.: New galactic open cluster candidates, Online Material p 16
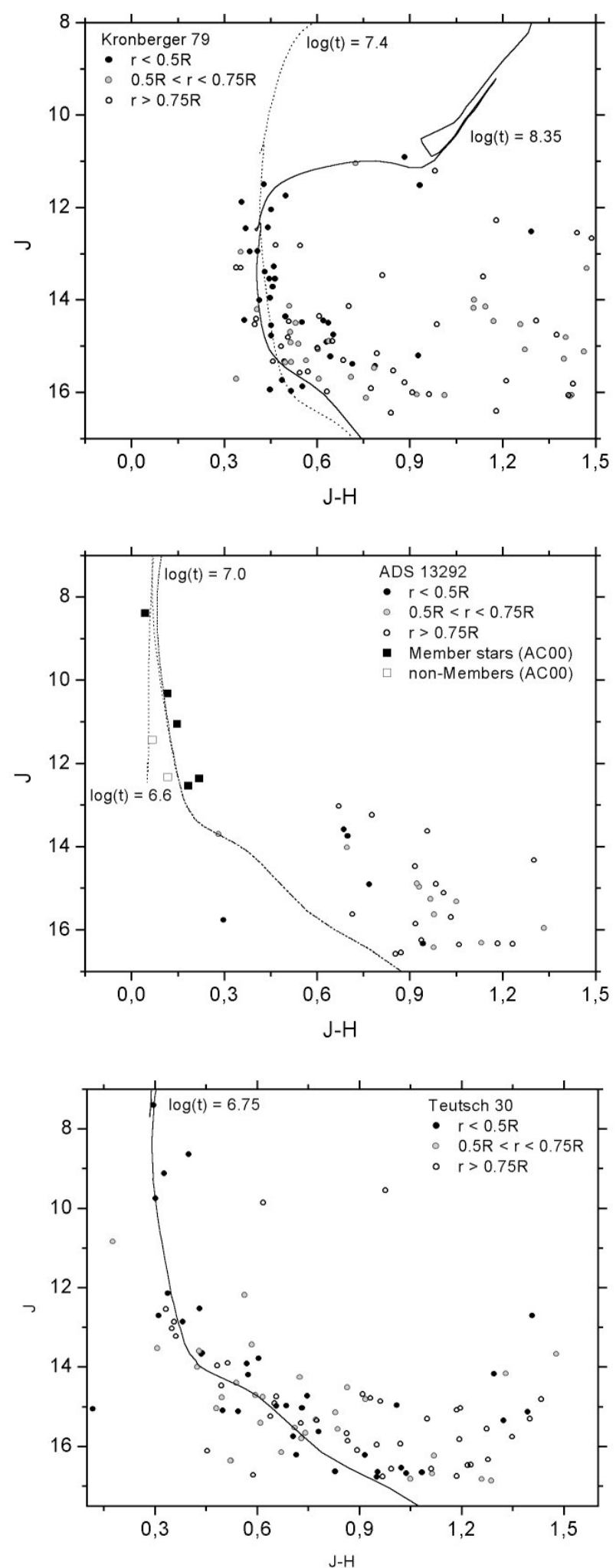

Fig. 7. continued. 
M. Kronberger et al.: New galactic open cluster candidates, Online Material p 17
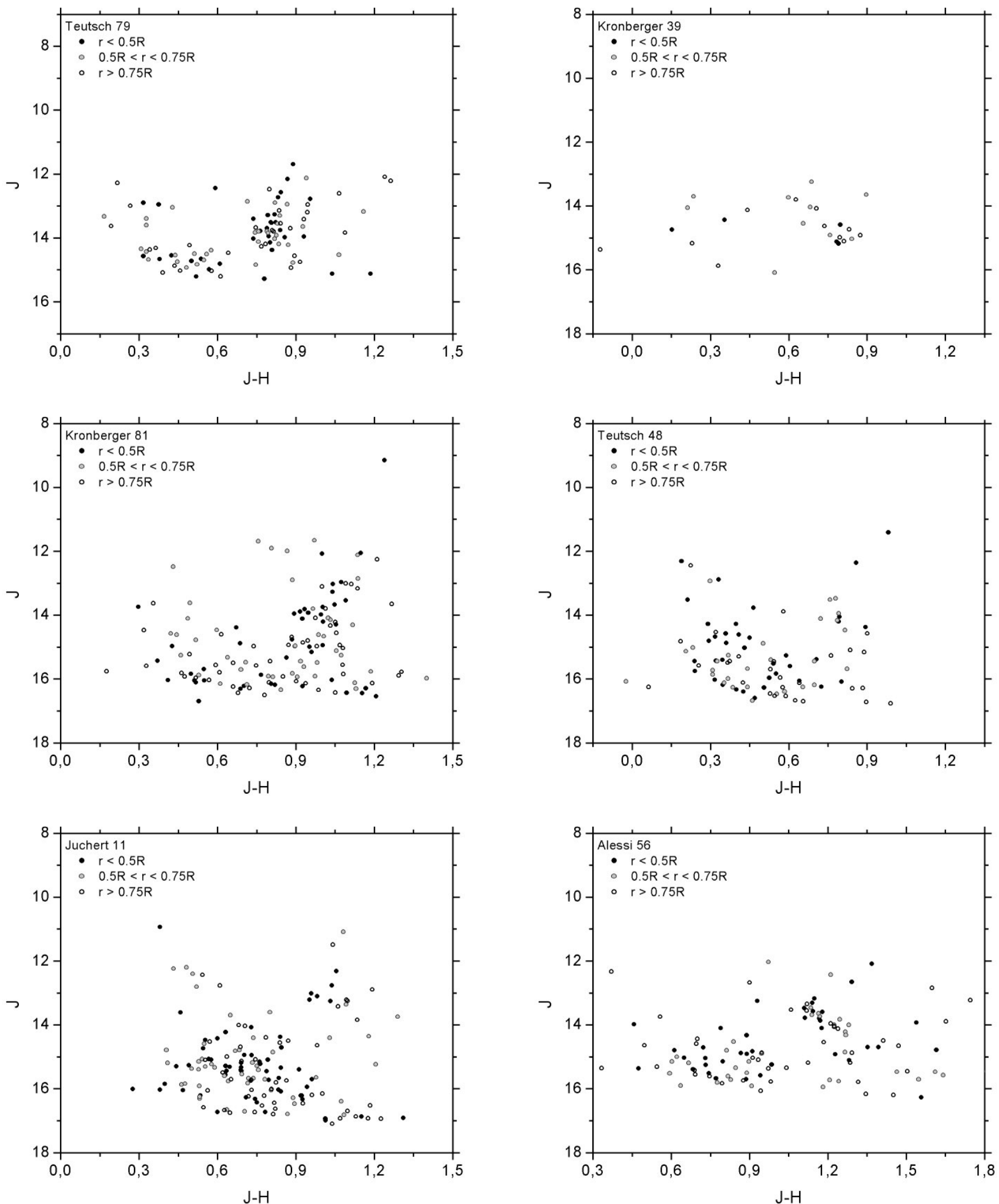

Fig. 8. CMDs of the cluster candidates presented in Table 2b. Each diagram contains only those stars with a distance from the cluster center that is less than the visual cluster radius $R$ and with $J$ and $H$ magnitudes derived either via aperture photometry $\left(r d_{-} f l g=1\right)$ or via point-spread function fitting $\left(r d_{-} f l g=2\right)$.

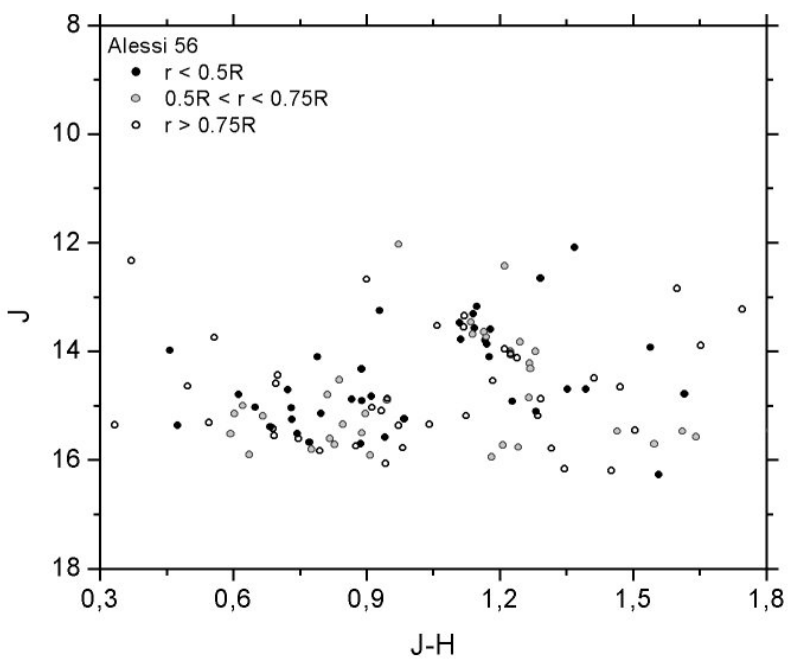

Fig. 8. continued. 
M. Kronberger et al.: New galactic open cluster candidates, Online Material p 18
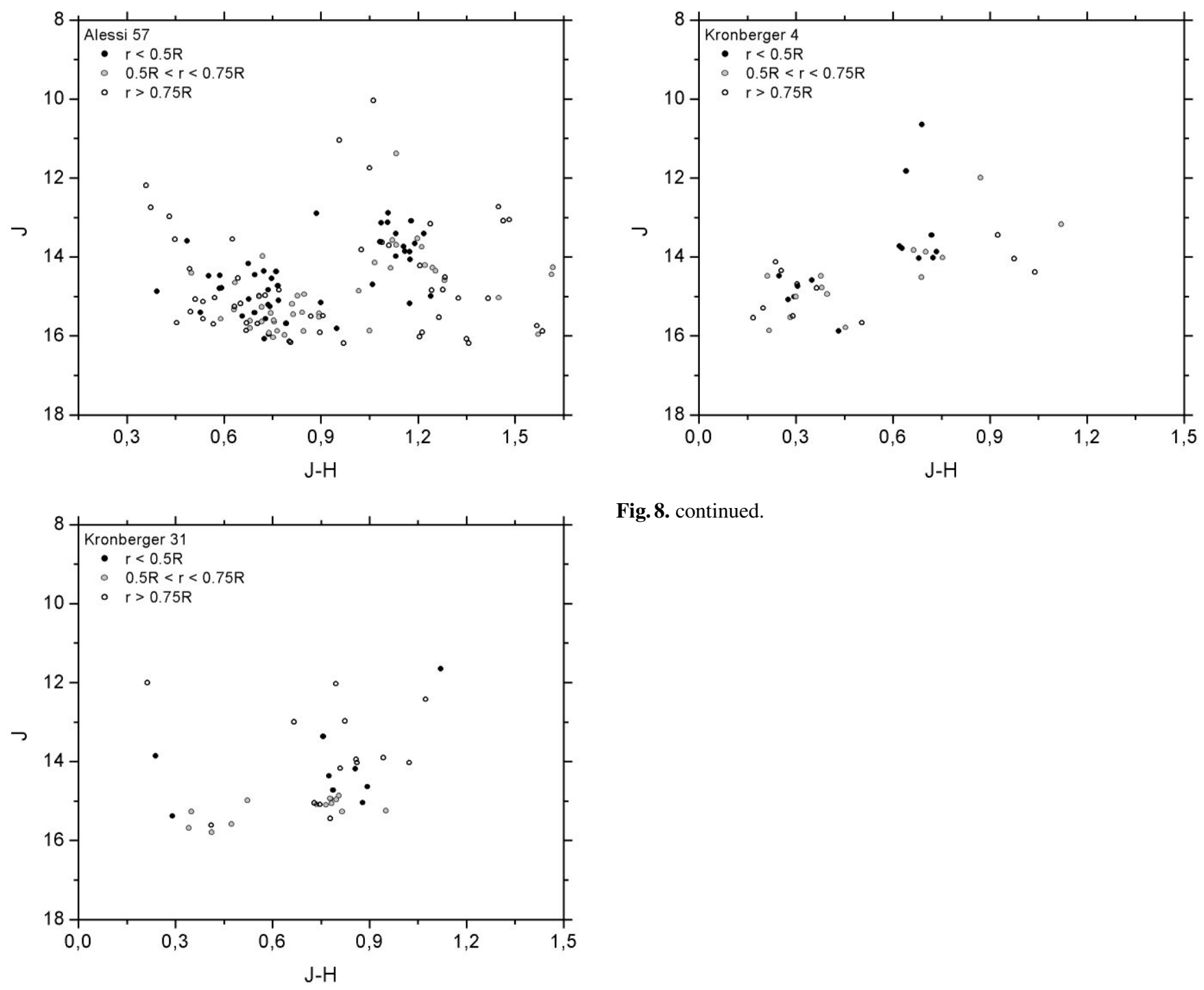

Fig. 8. continued.

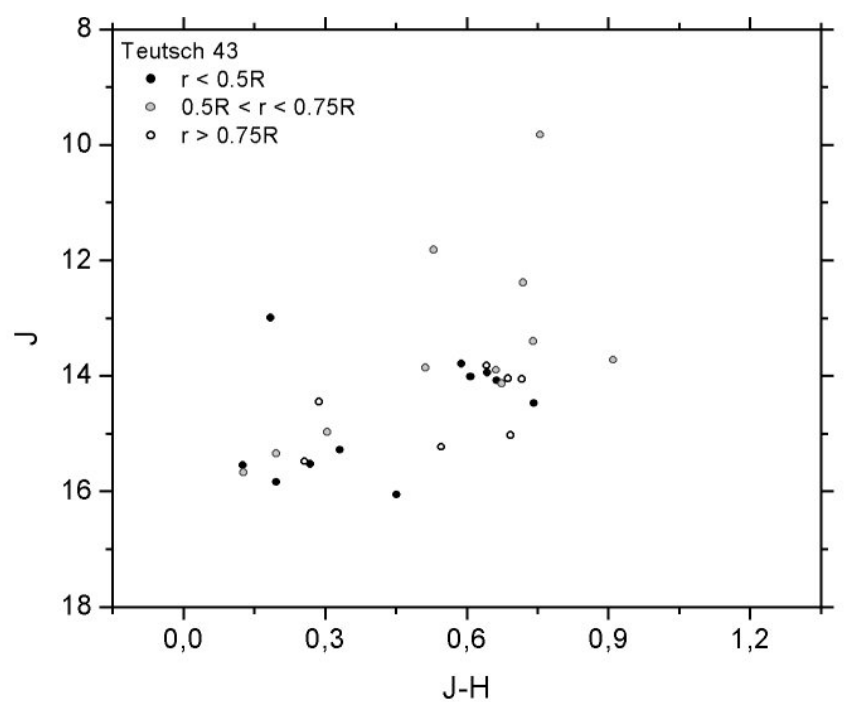

Fig. 8. continued. 
M. Kronberger et al.: New galactic open cluster candidates, Online Material p 19
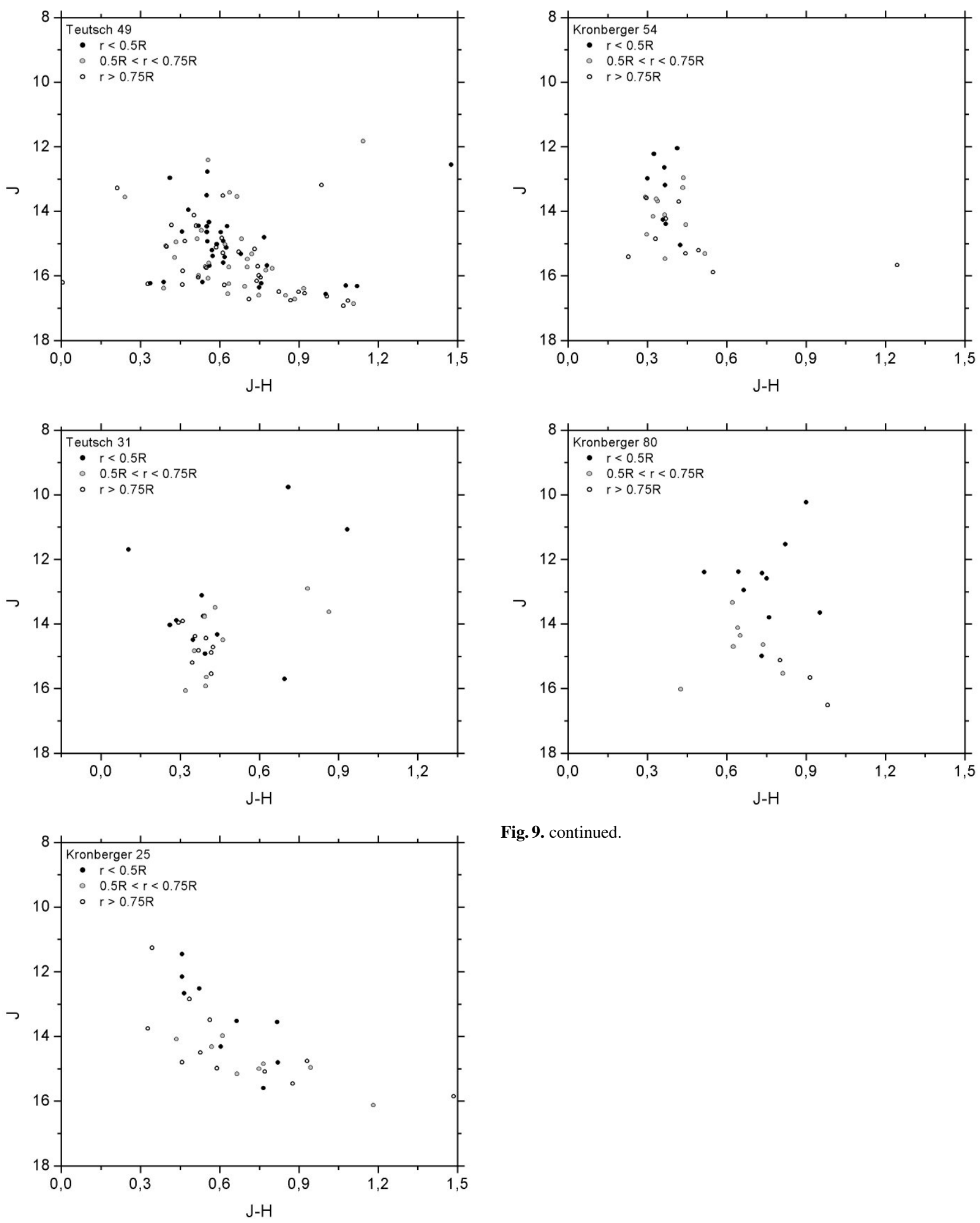

Fig. 9. continued.

Fig. 9. CMDs of the cluster candidates presented in Table 2c. Each diagram contains only those stars with a distance from the cluster center that is less than the visual cluster radius $R$ and with $J$ and $H$ magnitudes derived either via aperture photometry $\left(r d_{-} f l g=1\right)$ or via point-spread function fitting $\left(r d \_f l g=2\right)$. 
M. Kronberger et al.: New galactic open cluster candidates, Online Material p 20
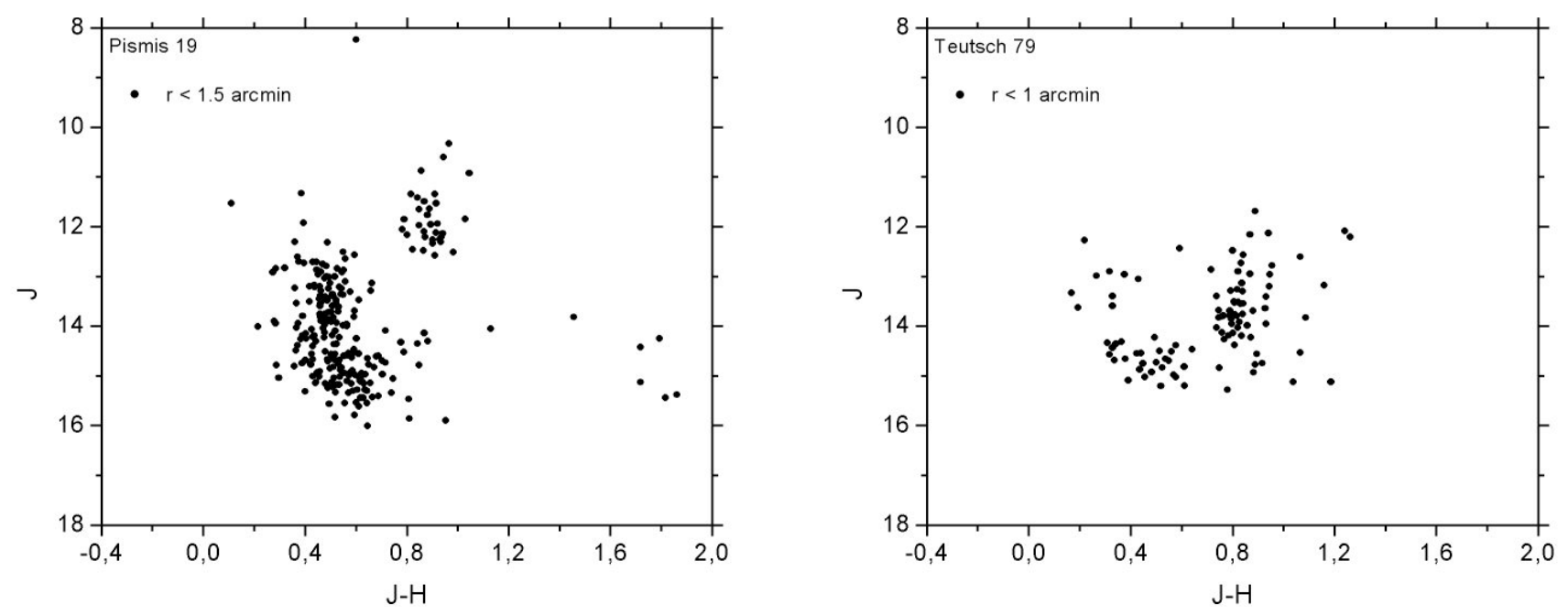

Fig. 10. Comparison of the CMDs of Pismis 19 (left) and Teutsch 79 (right). Both diagrams contain only those stars with $J$ and $H$ magnitudes derived either via aperture photometry $\left(r d_{f} l g=1\right)$ or via point-spread function fitting $\left(r d_{f} l g=2\right)$. Extraction radii of $1.5^{\prime}($ Pismis 19$)$ and $1^{\prime}$ (Teutsch 79) were taken.
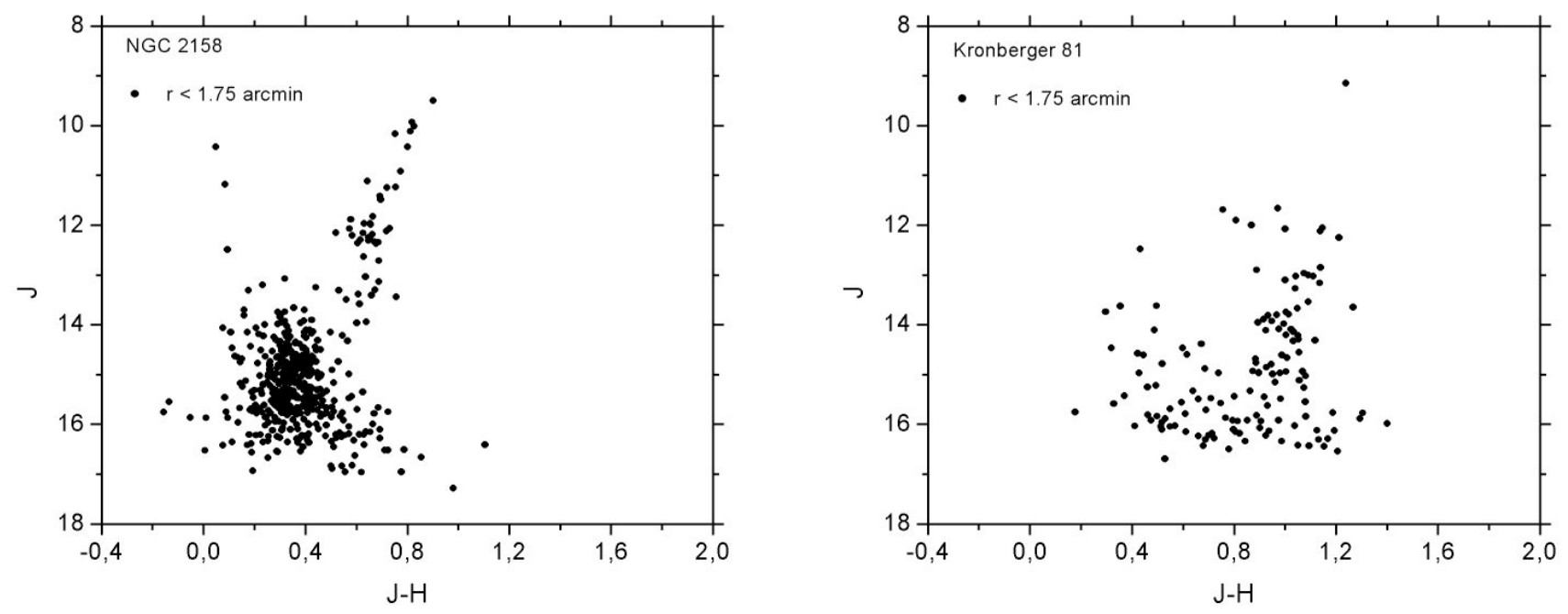

Fig. 11. Comparison of the CMDs of NGC 2158 (left) and Kronberger 81 (right). Both diagrams contain only those stars with $J$ and $H$ magnitudes derived either via aperture photometry $\left(r d_{f} l g=1\right)$ or via point-spread function fitting $\left(r d_{f} l g=2\right)$. In each case, an extraction radius of $1.75^{\prime}$ was taken. 